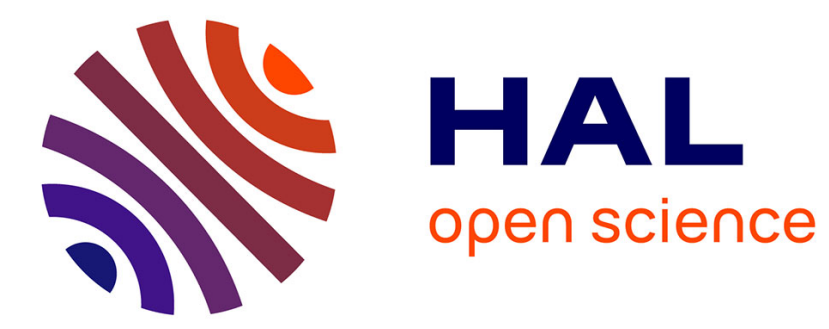

\title{
Université Paris 1 Panthéon-Sorbonne
}

Rapport Hcéres

\section{To cite this version:}

Rapport d'évaluation d'un établissement. Université Paris 1 Panthéon-Sorbonne. 2018. hceres02026327

\section{HAL Id: hceres-02026327 https://hal-hceres.archives-ouvertes.fr/hceres-02026327}

Submitted on 20 Feb 2019

HAL is a multi-disciplinary open access archive for the deposit and dissemination of scientific research documents, whether they are published or not. The documents may come from teaching and research institutions in France or abroad, or from public or private research centers.
L'archive ouverte pluridisciplinaire HAL, est destinée au dépôt et à la diffusion de documents scientifiques de niveau recherche, publiés ou non, émanant des établissements d'enseignement et de recherche français ou étrangers, des laboratoires publics ou privés. 
Éva luation des éta b lissements

RAPPORTD'ÉVALUATION DE L'UNIVERSITÉ PARIS 1 PANTHÉONSORBONNE

CAMPAGNE D'ÉVALUATION 2017-2018 VAGUE D 
Pourle Hcéres ${ }^{1}$ :

Michel Cosnard, Président
Au nom du comité d'experts:

Philippe Baudorre, Président du comité

En vertu du décret ${ }^{\circ} 2014-1365$ du 14 novembre 2014 :

${ }^{1}$ Le président du Hcéres « contresigne les rapports d 'évalua tion établis par les comités d'experts et signés par leur président. » (Artic le 8 , a linéa 5). 


\section{SOMMAIRE}

I. Présentation de l'établissement............................................................................................. 6

II. Le positionnement institutionnel et la stratégie de développement de l'établissement... 8

1/ Une stratégie d'alliance a mbitieuse mais aux contours encore flous.............................................................

2 / Un projet stra tégique à préciser et des partenaria ts à mieux exploiter.......................................................9

III. La gouvemance et le pilotage de l'établissement........................................................... 11

1 / Une organisation fonctionnelle en forte évolution mais une synergie entre structures de formation et de recherche rendue difficile par la dispersion des sites.........................................................................................11

2 / Une gouvemance complexe mais équilibrée ........................................................................................12

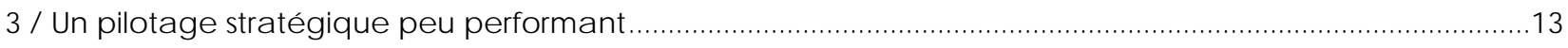

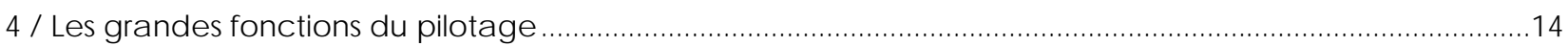

a / Une prise de conscience des besoins en outils de pilotage pour alimenter une réelle stra tégie politique en matière de ressources huma ines.....................................................................................................14

b / Une situation financière vulnérable, a dossée à des outils de pilotage trop rudimentaires et fragilisée par les opérations immobilières en cours.

c / Des projets immobiliers largement engagés, porteurs d'avenir pour l'université, dont il convient d'assurer

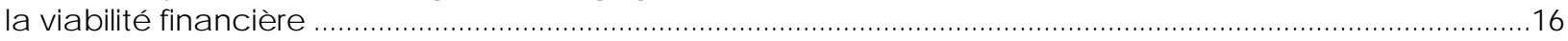

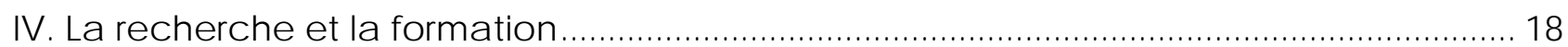

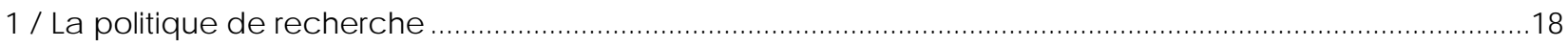

a / Une struc turation complexe ma is globalement effic ace des ac tivités de recherche.................................18

b / Une politique qui soutient l'excellence malgré le défic it des outils de suivi des activités de rec herche ..18

2 / La politique de formation initiale et continue ..................................................................................................19

a / Des fo mations no mbreuses, fortes et origina les....................................................................................20

b / Des diffic ultés liées à l'a ttra c tivité ...............................................................................................................20

c / Une CFVU au cœur du dispositif, organe de contrôle et d'échanges.........................................................21

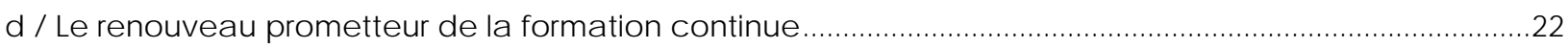

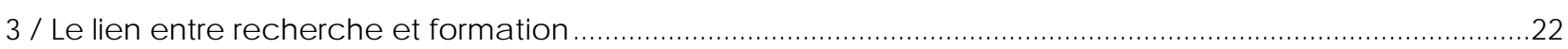

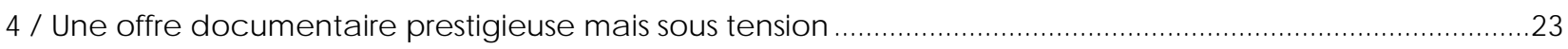

a / Risque de décrochage de la documentation pour la recherche ..............................................................23

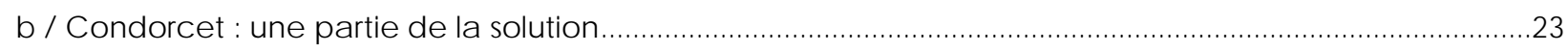

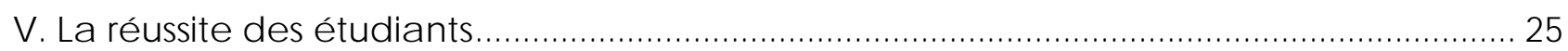

$1 /$ Un dialogue effic a ce entre étudiants et composantes........................................................................25

2 / Une politique de vie étudiante dynamique, grâce à ses asso cia tions et ses servic es...................................26

VI. La valorisation et la culture scientifique .............................................................. 28

$1 /$ Des résultats de la recherche insuffisa mment va lorisés...............................................................................28

2 / Des dispositifs de diffusion modestes a u regard du potentiel de l'éta blissement.........................................28

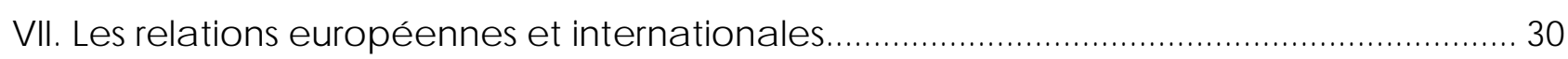

1 / Restructuration prometteuse de la direction des relations intemationales....................................................30

2 / Une politique RI fondée sur les demandes des composa ntes....................................................................30

3 / Développer la mobilité et en améliorer l'accompagnement......................................................................30

4 / Déloc alisation des formations et insertion dans des réseaux : une force de Pa ris 1.....................................31 


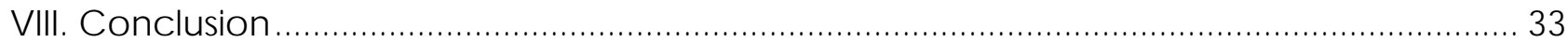

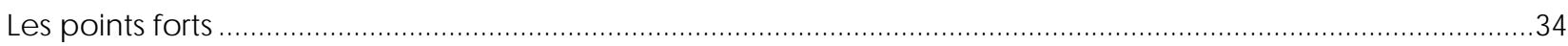

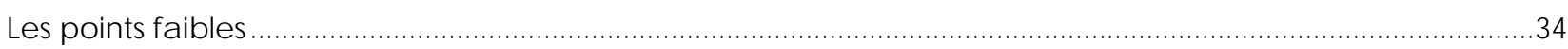

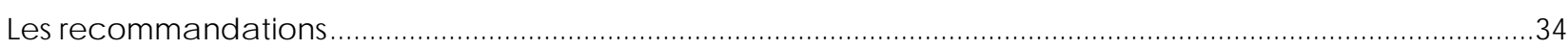

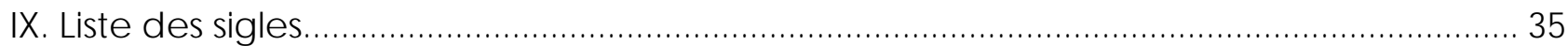

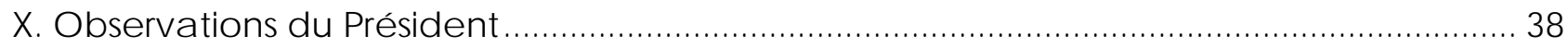

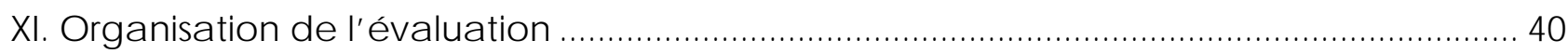




\section{PRESENTATION DE L'ETABLISSEMENT}

L'université Paris 1, dite «Panthéon-Sorbonne ${ }^{\circledR}$, a été créée en 1971, lors de la division de l'université de Paris en treize établissements autonomes. Elle intègre alors ${ }^{2}$ une partie des enseignements dispensés au sein de deux des quatre facultés parisiennes, la faculté de droit et dessciences économiques (Panthéon) et la faculté des lettres et sciences humaines (Sorbonne) a ssociant ainsi, dès sa c réation, plusieurs champs disciplinaires : le droit, l'économie, les sciences humaines (géographie, histoire, philosophie et histoire de l'art) et les sciences sociales ${ }^{3}$. Elle connaît alors pendant plusieurs décennies une forte augmentation de ses effectifs et un développement de ses composantes a insi que de ses sites ${ }^{4}$.

Aujourd'hui, l'effectif global d'étudiants inscrits dans des diplômes nationaux est réparti de façon équilibrée entre les tro is principaux domaines - 9750 en sciences économiques et gestion, 11600 en sciences humaines et sociales, 10300 en sciences jurid iques et sciences politiques - au sein de 10 UFR, auxquels il faut ajouter 620 inscrits dans les six instituts de l'établissement 5 . Au total, en 2016/2017, 42343 étudiants sont insc rits à Paris 1 , dont 37853 étudiants en diplômes nationa ux (licence, lic ence professionnelle ou master) et 4490 étudiants en diplômes universitaires (DU), ma gistères ou préparations (a grégation, concours). Paris 1 a également accueilli 2730 doc torants et 40 HDR, 246 Erasmus, 511 étudiants en programmes d'échanges et plus d'une centaine d'étudiants inscrits dans le parcours d'intégration des refugiés. Ces effectifs sont, depuis plusieurs années, stabilisés.

L'offre de formation de Paris 1 se répartit en 13 mentions de licence (et 42 parcours), 4 licences professionnelles et 64 mentions de master. Les étudiants ont la possibilité de suivre 16 doubles licences pluridisciplinaires. La recherche est portée par 36 équipes de recherche dont 24 UMR, en cotutelle avec le C NRS, I'IRD ou l'Inra, et 12 EA (contre 19 dans la précédente évaluation) et peut s'appuyer sur un fort potentiel de «plus de 1000 enseignants-cherc heurs et chercheurs $»$.

Paris 1 emploie 2614 personnes qui se répartissent ainsi : 1200 Biatss ( 804 fonc tionnaires et 396 contractuels) et 1414 enseignants (814 fonctionnaires et 600 contractuels). Les enseignants sont pour 722 des enseignants chercheurs et 92 des enseignants du second degré. Paris 1 héberge également de nombreux personnels relevant d'EPST: 691 personnels hébergés dont 637 catégorie A (principalement CNRS, 429, et INRA, 53), 48 catégorie B (dont CNRS 43) et 6 catégorie C (dont 5 CNRS).

L'établissement compte 10 écoles doc tora les qui participent à un collège doctoral commun.

Par ses effectifs et ses moyens, Paris 1 est donc une université sinon «de masse » du moins de grande envergure, tout en ma intenant une exigence d'exc ellence.

Passée aux «responsa bilités et compétenc es éla rgies 》 (RCE7) au 1 ja nvier 2011, Paris 1 a connu des diffic ultés budgétaires en 2012 et 2013 puis a réussi à rééquilibrer ses exercices. Son budget est actuellement de $213 \mathrm{M€}$ (Bl 2018), 173,3 en dépenses de personnel, 36,1 en fonc tionnement et 3,6 en investissement.

Une des caractéristiques historiques de Panis 1 est le nombre et la dispersion de ses sites (26 sites pour une superficie totale de $90000 \mathrm{~m}^{2}$ ). Les principales implantations sont dans le 5 ème et le 13 ème arrondissements de Paris. Deux importants projets d'aménagement, ceux de Lourcine, dont la livraison a été retardée, et de Condorcet, vont permettre à Pa ris 1 , à court et moyen terme, d'acc roître ses surfaces et de réaménager ses implantations. Ces nouveaux bâtiments répondront à la pénurie de surfaces dont Paris 1 souffre de façon récurrente car elle disperse ses équipes de recherche, pèse sur ses activités pédagogiques et grève son budget.

1 Cf. PV du CA du 14 septembre 2017 : «La dénomination «Paris 1 Panthéon-Sorbonne »n'étant à ce jour fomalisée que par nos seuls statuts, le Président a offic iellement saisi la nouvelle Ministre de l'Enseignement supérieur, de la Recherche et de l'innovation afin que la reconnaissance de ce nom soit consacrée juridiquement et que soit garanti à l'établissement l'usage du nom «Sorbonne ». La réponse de la ministre est positive et a été transmise au Rectorat, ce qui ouvre la voie à la public ation à venir de la modification du décret demandée. Cette interpellation faisait suite à la reconnaissance par décret de l'usage du nom «Sorbonne » par l'établissement né de la fusion entre les universités Paris 4 et Paris $6 »$.

Déc rets des 8 oc to bre et 23 déc embre 1970.

Décret $n^{\circ} 70-246$ du 21 mars 1970 relatif à la mise en place des universités.

Cf. les rapports AERES de 2010 et 2013, en ligne.

Données foumies parle tableau «En chiffres » présenté sur le site de Paris 1.

Rapport d'auto-évaluation (RAE) p.15.

Disposition de la loi relative aux libertés et aux responsabilités des universités du 10 a oût 2007 conférant aux universités des compétences budgétaires (autonomie budgétaire) et de gestion des ressources huma ines (recrutement d'agents contractuels et gestion desprimes par le président, répartition des obligations de service et création de dispositifs d'intéressement par le conseil d'administration. Voir Code de l'éducation, artic le L712-8. 
En devenant, en 2010, membre fondateur du Pôle de recherche et d'enseignement supérieur (Pres) Hésam (Hautes études - Sorbonne - Arts et Métiers), Paris 1 a vait «dû réorienter sa stratégie ». Le départ de plusieurs membres fondateurs du Pres $^{9}$ a précédé la transformation de celui-ci en Comue Hésam en août 2015. Paris 1 a alors de nouveau revu sa stratégie. L'équipe présidentielle élue au printemps 2016 a ouvert au sein de l'établissement une consultation qui s'est conclue par le vote en CA en novembre 2016 du retrait de la Comue Hésam, retrait effectif en mai 2017. Une nouvelle politique d'alliance était en cours d'élaboration au moment de la visite du comité.

Les évaluations AERES de 2010 et 2013 avaient conduit à des préconisations convergentes insistant tout particulièrement sur la nécessité de réfomes structurelles et sur la mise en place d'outils et de procédures de gestion permettant une meilleure maîtrise de l'équilibre budgétaire. Il était conseillé un meilleur pilotage de l'offre de formation permettant d'assurer sa soutenabilité financière, l'amélioration de l'accueil, de l'intégration et du suivi des étudiants diplômés et une meilleure communication exteme pemettant de promouvoir la «marque »Panthéon-Sorbonne.

Le rapport 2013 so uligna it plus partic ulièrement «les deux c ontra intes fortes qui pèsent sur l'éta blissement : une situation financière tendue et une situation immobilière préoccupante »(p. 30).

Ces préconisations restent des points d'attention au cœur de la présente expertise : Paris 1 est-elle en mesure d'évaluer et de maîtriser ses coûts, compte tenu de sa taille, de la dispersion de ses sites et de son mode d'organisation «fédérateur»? Pour répondre à cette question, le comité a identifié quelques thèmes majeurs:

- Le mode d'organisation de l'établissement ;

- La stratégie d'alliances et de positionnement;

- La politique dans le domaine de la recherche et de la fomation (définition, validation, mise en œuvre) ;

- La politique de partenariats avec le monde socio-économique et d'intemationalisation

Il a souhaité éga lement évaluer la place de l'étudiant au sein de l'établissement.

Rapport AERES 2013, p. 7.

L'École des hautes études en sciences sociales, l'École nationale des chartes, l'École pratique des hautes études, la FSH et I'Inalco. 


\section{LE POSITIONNEMENT INSTITUTIONNEL ET LA STRATEGIE DE DEVELOPPEMENT DE L'ETABLISSEMENT}

Principal pôle universitaire français dans le secteur des sciences humaines et sociales (SHS), consciente de son excellence dans la plupart des champs disciplinaires qui la constituent ${ }^{10}$, l'université Paris 1 souhaite fédérer des partenaires nationaux ou intemationaux autour de projets communs mais veut éviter toute formule d'alliance ou d'intégration trop formalisée dans laquelle elle craint de ne pouvoir exprimer son potentiel voire risquerait, selon elle, de perdre son identité. Si les documents rassemblés en vue de cette évaluation ${ }^{11}$ restent disc rets sur les perspec tives stratégiques à long teme, c'est que Paris 1 est déterminée à maintenir ce qu'elle est et représente, bien plus que préoccupée de définir ce qu'elle poura it être. Trois éléments contribuent à cette forte image de soi : la qualité reconnue de la recherche et de la formation, la diversité des disciplines au sein des SHS et leur taille, celle-ci n'étant jamais perçue comme un obstacle à l'excellence mais au contraire comme sa condition nécessaire. Ces éléments permettent de comprendre ses choix d'alliance, de partenariat et de positionnement.

\section{1/ UNE STRATEG IE D'AШIANCE AMBITEUSE MAIS AUX CONTO URS ENC ORE FLO US}

Le RAE note sans ambages que la tentative d'inscrire Paris 1 Panthéon-Sorbonne dans une Comue fut un «échec »12. Moins d'un an après la sortie effective de la Comue Hésam en mai 2017, cette rupture est devenue un état de fait qui n'est plus débattu ni contesté. Le rapport AERES 2013 voyait dans le Pres Hésam «un facteur particulièrement important pour renforcer la place de l'établissement dans le paysage universitaire français, européen et mondial » ainsi qu'un « levier de mutualisation des tâches et une source potentielle d'économies». Mais les évaluateurs avaient bien senti que cette analyse n'était pas partagée au sein de l'université, puisqu'ils ajoutaient: «le Pres Hésam semble encore perçu au sein de la communauté universitaire comme un corps étranger qui absorbe tout a u plus des ressources mais ne permet pas réellement d'améliorer la situation de la formation ou de la recherche ». Le faible succès aux PIA 1 et $2^{13}$, la sortie du Pres de plusieurs membres fondateurs, le fait que Paris 1 so it la seule université dans ce qui était devenu en août 2015 une Comue, la disproportion entre la taille de Paris 1 et sa représentation dans les instances de gouvemance de celle-ci, ne pouvaient transformer en adhésion ce qui n'avait été, dès l'origine, qu'un choix par défaut ${ }^{14}$. Le retrait de Paris 1 de la Comue s'est effectué à l'issue d'un large débat au sein de l'établissement. Un congrès élargi aux directeurs de composantes et d'équipes a été réuni le 16 novembre 2016 en présence du président de la Comue Hésam, et de son délégué général. Le 17 novembre 2016, le CA votait le principe de la sortie de la Comue Hésa $m^{15}$, devenue effective quelques mois plus tard.

Selon une formule régulièrement reprise par le président et son équipe, «l'Université Paris 1, PanthéonSorbonne forme une «véritable Comue » qui fonctionne, de manière efficace, depuis 197016. 》 Elle en a la taille, la visibilité, l'ouverture disciplinaire. Elle cherche désomais une voie «expérimentale », d'association ou d'alliance, formalisée mais souple, qui ne s'inscrirait pas dans les trois options définies par la loi du 22 juillet 2013. L'équipe actuelle envisage la création d'un Groupement d'Intérêt Universitaire ${ }^{17}$, sans chef de file, «Sorbonne Alliances», qui s'articulerait avec une confédération européenne d'universités. \| s'agirait de donner naissance à «un modèle institutionnel innovant (...), aussi souple et léger que possible, sans superstruc ture concurrençant les étab lissements fédérés, et fonctionna nt avant tout sur projets »18.

Mais si cette stratégie d'alliances et d'associations est ambitieuse, si elle est cohérente et dénote une réelle volonté d'ouverture à d'autres établissements, ses contours paraissent encore flous, notamment en temes juridiques et financiers. Au moment de l'évaluation, Paris 1 pouvait faire état d'un accord avec l'ESCP, de contacts avancés avec I'Ined et l'INHA. Des échanges sur ce point avec l'université Paris 2 ont été évoqués

RAE, p. 44.

RAE, axes stratégiques, présentation du président.

RAE, p. 6.

13 Le programme «Paris Nouveaux Mondes » présenté par le Pres Hésam n'avait pas obtenu en 2012 le label d'Idex mais un soutien financier équivalent à la moitié du montant demandé car jugé «prometteur», d'où l'expression de «demiIdex» (RAE, p. 7 et 20). L'action a été conduite en 2012-2016 ma is a souffert des diffic ultés intemes au Pres. Les projets portés par Hésam dans la soumission de projets au PIA 2 n'ont été retenus ni dans le cadre de l'Idex ni dans celui de l'ISite.

14 Auparavant, Paris 1 s'était engagée dans la constitution d'un Pres dès 2006 au côté de Paris III et Paris V ma is s'en est trouvée exclue en 2009.

15 Par 18 voix pour, 6 voix contre et 9 abstentions.

16 RAE p. 16.

17 II n'existe aucune forme juridique de cette nature ; c'est un concept proposé par Paris I qui s'inspirerait du Groupement d'intérêt économique ; cf. Axes stratégiques 2010-2023, p.6.

18 Ibid. Le CA du 22 décembre 2017 a voté la poursuite de disc ussions sur la base d'un «système souple et non fusionnel ». 
en CA de même ${ }^{19} q$ qu$^{\prime}$ a été a vancée la volonté de s'associer avec une université scientifique étrangère, intégrant des champs disc ip lina ires aujourd'hui non représentés a u sein de Paris 1.

Paris 1 oppose, dans son RAE, la sortie de la Comue Hésam, considérée comme une impasse, à «l'intégration consolidée dans le campus Condorcet», véritable ouverture en termes d'alliances et de partenariats fruc tueux. La création d'une «C ité des humanités et des sciences sociales » sur les sites d'Aubervilliers et de La Chapelle est un projet ancien, inscrit dans le plan Campus lancé par l'État depuis $2008^{20}$. L'établissement public a été créé en février 201721. La première tranche de bâtiments sera livrée en juin 2019 ; le Grand équipement documentaire et le transfert d'unités de recherche, nota mment celles de l'EHESS, seront effectifs au printemps 2020. Pour le site de La Chapelle qui sera essentiellement occupé par Paris 1, il faudra attendre 2022. Comme on le verra dans d'autres chapitres du présent rapport, Paris 1 entretient d'excellentes rela tions avec le CNRS, I'IRD et l'Ined. Le futur Campus Condorcet peut donc être un terrain d'expérimentation favorable à ces nouveaux rapprochements. Ainsi, avec le CNRS, I'EHESS, I'ENC, I'EPHE, la FMSH, I'Ined, Paris I, Paris III, Paris VIII, Paris X et Panis XIII, «le Campus Condorcet réunit onze membres de forte notorété intemationale, unis de longue date par d'étroites relations scientifiques et pédagogiques ${ }^{22}$. » Paris 1 et ses partenaires veulent faire de ce Campus Condorcet un leader en sciences humaines et sociales en Europe, avec l'ambition de rivaliser avec la London School of Economics and Politic al Science (LSE). Toutefois, si Condorcet va permettre d'accroître les surfaces (surtout à partir de 2022) et offrira des possibilités de collaborations nouvelles avec des universités et des organismes, le projet ne constitue pas une véritable altemative à une stratégie d'alliances plus globale.

\section{2 / UN PRO J ETSTRATEG IQUE A PRECISER ETDES PARTENARIATS A MIEUX EXPLOITER}

Le cap est clair: Panis 1 souhaite «réussir à demeurer tout à la fois une université de recherche de rang mondial et une université de formation d'excellence pour le plus grand nombre ${ }^{3}$. Mais, à l'échelle de l'établissement, les objectifs plus opérationnels permettant d'y parvenir, manquent. Les développements stratégiques rapprochés, en matière de formation et de recherche, étant laissés à l'initiative des composantes (UFR et équipes), l'objec tif collectif le plus clairement identifiable est aujourd 'hui de développer les espaces et d'accroître les ressources.

À l'échelle intemationale, l'université a ffiche de grandes ambitions, capitalisant notamment sur le prestige de la marque "Sorbonne». Outre ses alliances déjà anciennes avec l'université new-yorka ise Columbia ou l'école européenne ESCP Europe, Paris 1 entend construire une confédération d'établissements européens, à l'image des liensqui l'unissent de longue date à l'université madrilène Complutense.

De même, Paris 1 s'ouvre au monde de l'entreprise et du tissu socio-économique, grâce à de nombreuses modalités de partenariat (salons professionnels, interventions de cadres d'entreprises, stages et prérecrutement en niveau master, etc.).

Mais si les a mbitions sont très cla irement affichées, les liens réels avec les entreprises sont enc ore peu resserés et les expériences concrètes (comme les bourses BNP Paribas) encore trop rares. En effet, il manque à l'université une politique globale de partenariats à même de rapprocher davantage les SHS et le monde des entreprises. Le comité a constaté que la cellule chargée du lien avec les entreprises au sein de la «direction partenariat entreprises et insertion professionnelle »(DPEIP) est déterminée et résolue mais encore trop isolée, pas suffisamment mise en avant au cœur de la stra tégie de l'université. Paris 1 pourrait par exemple mettre en place des campagnes systématiques pour inciter les enseignants à faire venir leurs étudiants aux diverses manifestations avec les milieux soc io-économiques.

À cet égard, les collectivités teritoriales, partenaires institutionnels de l'université, - la Région île-de-France et la Ville de Paris - ont une vision positive et dynamique de l'engagement de Paris 1 dans le tissu socioéconomique. À moyen et long teme, elles attendent de Paris 1 qu'elle puisse répondre encore mieux-grâce à sa politique de formation continue - aux besoins du marché de l'emploi. Si cette intention stratégique est visible dans la politique de Panis 1 , il faudra, au-delà de la formation d'étudiants jusqu'à l'âge de 25 ans, que l'université contribue à répondre aux problématiques du chômage et de l'obsolescence des compétences. Ces collectivités temitoriales se félic itent du retour très positif d'acteurs socioprofessionnels sur les formations

$19 \quad$ PV du CA du 12 octobre 2017

20 Déjà abordé dans les rapports AERES de 2010 et 2013

21 Décret statutaire publié en décembre 2017. Les membres de l'établissement sont le Centre national de la recherche sc ientifique (CNRS), l'École des hautes études en sciences sociales (EHESS), l'École nationale des chartes (ENC), l'École pratique des hautes études (EPHE), la Fondation Maison des sciences de l'homme (FMSH), l'Institut national d'études démographiques (Ined), I'université de Paris I "Panthéon-Sorbonne", l'université de Paris III "Sorbonne nouvelle", I'université de Paris VIII 'Vincennes à Sa int-Denis", I'université Paris X "Pa ris-Nanterre", I' université de Paris XIII "Paris-Nord".

22 Source : site intemet Campus Condorcet.

23 RAE, p.1. 
proposées a ujourd'hui par Paris 1, notamment en management et ressources humaines. La région lle-deFrance comme la ville de Paris, soutiennent avec force les projets Lourcine, Condorc et-Aubervilliers et La Chapelle. 


\section{LA GOUVERNANCE ET LE PILOTAGE DE L'ETABLISSEMENT}

Les nombreuses structures et implantations d'un établissement de la taille de Paris 1 ainsi que la diversité de ses formations et de ses doma ines de recherche constituent un élément de complexité non négligeable pour l'organisation inteme a insi qu'un environnement peu favorable à la diffusion d'une information pertinente et vraiment partagée. Historiquement, l'établissement a privilégié deux principes d'organisation, la subsidiarité et le respect de l'égalité de chaque composante dans la participation à la gestion commune. Maintenir ce qui est présenté et ressenti en inteme comme l'identité, l'esprit de Paris 1, suppose cependant que l'on dispose d'outils de communic a tion performants, au service d'un projet commun clairement affic hé.

\section{1 / UNE ORGANISATIO N FONCTIONNEUE EN FO RTE EVOLUTION MAIS UNE SYNERG IE ENTRE STRUCTURES DE FO RMATION ETDE REC HERC HE RENDUE DIFFIC ILE PAR LA DISPERSION DES SITES}

Au fur et à mesure de la création ou du rattachement d'unités de formation et/ou de recherche, Paris 1 a dû recevoir en affectation, ac quérir ou louer de nouveaux bâtiments ${ }^{24}$. Ses activités se déploient a ujourd'hui sur 26 sites et une superficie de $90000 \mathrm{~m}^{2}$. Les principales implantations se trouvent dans le 5 ème (site historique du Panthéon, centre Broca, institut de Géographie) et le 13ème (centres Pierre Mendès-France et René Cassin, maison des sciences économiques) mais Paris 1 est aussi dans le 15ème (centre Saint-Charles), dans le 4ème (centre Mahler), dans le 6ème (centre de la rue du Four) ainsi qu'à Bourg-la-Reine, Nogent-sur-Mame et Nanterre 25 . En temes de surfaces ou de statut, le contraste est important entre le site historique du Panthéon (9 $700 \mathrm{~m}^{2}$ mis à disposition par l'État et la ville de Paris), le centre Saint-Charles (4 $900 \mathrm{~m}^{2}$ dont Paris 1 est affectataire) ou le centre Broca (2 $300 \mathrm{~m}^{2}$ que Panis 1 a acquis en 2009) et les $80 \mathrm{~m}^{2}$ des Éditions de la Sorbonne, rue Saint-J acques, qui ap partiennent à Paris 1.

Ces sites accueillent vingt-deux départements de formation ${ }^{26}$ parmi lesquels les 10 UFR statutaires, dont plusieurs ont changé de nom ${ }^{27}$, ma is également six instituts ${ }^{28}$, un centre de préparation aux carrères publiques (Cipcea), le département des langues, le service des sports et celui de la formation continue. Autant de composantes de tailles et de statuts très divers. À elle seule, par exemple, l'école de droit de la Sorbonne (UFR créée le 14 avril 2015 par la fusion des cinq UFR juridiques) accueille 13000 étudiants et plusieurs départements. La plupart de ces composantes sont sur deux voire qua tre sites pour certa ines (UFR d'histoire). Si la tendance consistant à renommer les UFR pour en faire des «écoles» ou des «départements»peut donner à ces composantes une visibilité nationale ou intemationale, elle brouille la structure statutaire et organisationnelle de Paris 1 telle qu'elle apparaît sur son site institutionnel et dans ses supports de communic ation. Par leur nombre, la diversité de leurs statuts, leurs multiples implantations, leurs effectifs, les unités de recherche ne composent pas un paysage plusfacile à appréhender (cf. infra).

Le transfert d'une douzaine de laboratoires en sciences humaines et sociales sur le campus Condorcet et de composantes sur le site Lourcine à l'été $2019^{29}$ et la perspective du transfert du 1er cycle sur le site de La Chapelle en 2022 pemettront de finaliser le resserrement des implantations amorcé depuis $2013^{30}$ mais vont profondément recomposer le paysage des différentes structures et seront à prendre en compte dans l'organisation future.

L'orga nigramme administratif a récemment évolué. Depuis 2016, la nouvelle équipe présidentielle, constatant un déficit d'informations fiables, a conduit une réorganisation profonde des grandes fonctions supports et un renouvellement de leur encadrement. Rattachés fonctionnellement à des vice-présidents ou chargés de mission composant le bureau de l'université, les pénimètres des servic es supports ont été cla rifiés, des fonctions ont été créées (direction de la communication, cellule d'aide au montage de projet), des services ont été fortement reconfigurés (formation continue), d'autres restructurés (DRH) même s'il reste encore à clarifier le positionnement de certains servic es (artic ulation de la cellule d'aide au pilotage avec les cellules de pilotage

24 Citons entre autres l'intégration du centre de recherches historiques et juridiques de la rue Malher en 1972, du centre Sa int-Charles pour les arts plastiques en 1973, du centre Tolbiac (aujourd 'hui centre Pierre Mendès-France) en 1973, du centre René-Cassin en 1990, de la maison des sciences économiques en 1998 ou du centre Broca.

25 Source : fiches intranet.

26 C'est l'intitulé qui est utilisé sur le site de Paris 1 sous l'onglet «Formation ».

27 CA du 14 décembre 2017, modification des statuts: I'UFR 02 devient «École d'économie de la Sorbonne »et l'UFR 04 «École desarts de la Sorbonne ».

28 Institut des assurances de Paris (IAP), Institut de recherche et d'études supérieures du Tourisme (Irest), Institut des sciences sociales du Travail (ISST), Institut du développement économique et social (IEDES), Institut de la démographie de I'université Paris 1 (Idup), l'institut d'études judicia ires «J ean Domat ».

29 SPSI p. 62 à 65 pour le détail des laboratoires et composantes transférés.

30 RAE p. 65 : passage de 29 à 26 sites et de 92000 à $91000 \mathrm{~m}^{2}$ entre 2013 et 2017. 
implantées au sein des directions des ressources humaines (DRH) et des affaires budgétaires (DAFB) par exemple). Le partage et l'appropriation de cette nouvelle organisation sont freinés par une communication inteme peu efficace pour laquelle un projet de refonte est en cours de réflexion. Il vise à fluidifier l'information au sein de l'organisation reconfigurée et permettre ainsi la visibilité de tous les services ${ }^{31}$.

Au niveau des composantes et dans le domaine de la recherche, l'information est partagée et la coordination est soutenue par des pratiques reconnues et appréciées (réunion mensuelle des directeurs de composantes sur un ordre du jour co-construit avec les partic ipants, conseil de la recherc he). En revanche, les fonctions sup ports et les servic es communs n'ont pas de temps d'éc hanges réguliers sur leurs ac tivités. Sur ce point, le format très large (près de 60 participants) de la réunion mensuelle entre la structure administrative autour de la directrice générale des senvices et les responsables administratifs des composantes, vécue comme une première étape utile, n'est pas, aux yeux du comité, suffisante pour assurer une infomation complète et fluide sur les a ctivités de chacun. Un besoin de réunions complémentaires, plus restreintes et plus thématiques, a été exprimé, traduisant le besoin réel de concertation et d'hamonisation, mais également la difficulté à les mettre en œuvre. Il serait souhaitable qu'une solution soit apportée tant ce besoin paraît au comité justifié.

\section{2 / UNE GOUVERNANCE COMPLEXE MAIS EQUILBREE}

Le renouvellement de l'équipe présidentielle au printemps 2016 s'inscrit dans la continuité des principes qui fondent l'équilibre institutionnel de Paris 1 et peuvent se résumer à deux exigences constamment affic hées: équilibre et éga lité. Elles se traduisent par un certain nombre de choix politiques, inscrits dans les statuts ou tacites mais présentés comme intangibles. De même que la présidence doit être assumée tour à tour par un représentant d'un des grands champs disciplinaires (principe dit «du toumiquet »), les vice-présidents des conseils vont par deux : un issu du collège $A$, un issu du collège $B$, avec un souci égal de représentation des grands domaines disciplinaires. La 1ère VP CA est en charge des affa ires institutionnelles en lien avec la DAJI ; elle prépare les CA et les réunions de directeurs de composantes; elle est chargée de dossiers stratégiques (la sortie de la Comue ou la mise en place des nouvelles formes d'alliance). La zème VP CA intervient dans le cadre de la pédagogie en altemance, des partenariats (avec la DPEIP) et de la soutenabilité de l'offre de formation; elle gère les comités de direction. Les 2 VP CFVU ont aussi chacun leur domaine propre : à l'une les formations, à l'autre la vie étudiante. La répartition entre les 2 VP recherche est moins tranchée, mais, pour tous, l'équilibre est supposé reposer sur l'échange et la confiance. Si l'on ajoute le VP étudiant du CA, membre du burea ${ }^{32}$, Paris 1 ne peut avoir que deux VP délégués ${ }^{33}$, les statuts limitant le nombre total de VP à 9. Cela apparaît au comité très insuffisant pour un établissement de l'importance de Paris 1 et conduit par exemple à confier un dossier aussi important que celui de l'immobilier à un enseignant-chercheur doté du simple statut de «chargé de mission ». Le comité propose donc de mettre un teme au «binôme de VP » ou d'augmenter statutairement le nombre des VP.

Les 16 VP et chargés de mission et un représentant Biatss constituent le bureau qui se réunit une fo is par mo is ${ }^{34}$ alors que le comité de direction se réunit chaque semaine ${ }^{35}$. La règle évoquée d'équilibre entre les champs disciplinaires et les collèges s'applique jusque dans la désignation des 16 membres composant le bureau et dans la composition des listes des candidats aux conseils. Une réunion des directeurs de composantes réunit mensuellement autour du président, les directeurs d'UFR, de départements et d'instituts.

Le rythme de réunion des conseils obéit à un calendrier fixé pour toute l'année (fonctionnement préc isé par l'article $62 \mathrm{du}$ règlement intérieur). Cette organisation témoigne de la volonté de donner aux conseils et commissions un rôle clé dans le fonctionnement politique de Paris 1 et contribue à une vie démocratique réelle et perçue comme telle au sein de l'établissement. Les conseils, qui accueillent de nombreux «invités», d'usage ou de circonstance, notamment les responsables de composantes, sont véritablement des lieux de débats, d'éc hanges, de prises de décision. Autour d'eux, les représenta nts élus orga nisent des consultations et assurent la diffusion des infomations. Les débats, tels que les rapportent les PV et d'a près les élus eux-mêmes,

\footnotetext{
Notamment pour l'aide aux personnels et a ux étudiants a ujourd'hui mal identifiés (médiateur, c ellule handicap).

Il y a un autre VP étudiant, celui du CAC.

Il s'agit du VP « ressources » et du VP RI.

34 Aux temes des statuts votés en février 2016: «Le bureau se compose de 16 membres au plus. II est présidé par le Président. »

Les membres du bureau sont élus au scrutin pluninominal par le Conseil d'administration sur proposition du Président de l'Université. Le membre étudiant et le membre des personnels de bibliothèque, ingénieurs, administratifs, techniciens, de service et de santé (Biatss) du bureau sont proposés par le Président parmi les membres de leur collège respectif du Conseil d'administration et dans le respect des résultats des élections universita ires. » (Sta tuts votés en février 2016).

35 Il existe, d'après le RAE, deux modalités de constitution du Codir : un «comité de direction élargi autour du DGS aux 3 directeurs généraux adjoints, à l'agent comptable et à l'ensemble des directeurs de service », et un Codir réunissant «l'équipe présidentielle formée des VP autour du président et du DGS ».
} 
sont riches et ouverts, parfois même trop longs et «bavards», particulièrement en CFVU où les propositions émanant des composantes sont présentées et débattues avant d'être amendées ou validées. Avec la volonté d'équilibre entre les collèges et les disciplines, le respect d'une forte autonomie des composantes reste bien au cœur de la gouvemance de Paris 1.

Mais pour poser les bases de ce projet commun et en assurer la mise en place et le suivi, le comité estime que Paris 1 doit disposer, notamment en matière de finances et de $\mathrm{RH}$, d'outils de pilotage partagés, entre directions et avec les composantes, les outils actuellement utilisés n'ayant pas les deux dimensions, infraa nnuelle et pluria nnuelle, nécessaires pour un pilotage efficace. Pa is 1 doit éga lement a méliorer des outils de communication inteme et exteme (mise en place d'une newsletter, d'un intranet et d'un environnement numérique de travail efficaces, réa ctivité de la page d'accueil en temes d'actualités) qui seront en mesure, sans nuire à la visibilité des composantes, d'assurer la présence et la cohérence de l'établissement lui-même. II ne s'agit pas seulement de construire une vitrine attractive mais un véritable espace de partage d'informations, accessible à tous et dans lequel tous peuvent se reconnaître.

\section{3 / UN PILOTAGE STRATEG IQ UE PEU PERFORMANT}

Cette volonté de partage et de transparence, se heurte en effet au déficit d'outils de gestion et d'indicateurs communs, à des vecteurs de communication inteme défaillants et à une structuration insuffisante des interac tions entre les servic es d'a bord puis entre les servic es et les composantes.

Au vu des distorsions des montants de masse salariale entre les bilans sociaux et les comptes financiers pour 2014 et $2015^{36}$, le décloisonnement des services porté par le projet «travailler ensemble à Paris 1 PanthéonSorbonne ${ }^{37} \mathrm{~s}^{\prime}$ imposait pour fluidifier le fonctionnement de l'université et assurer les bases de son pilotage. Aujourd'hui, les échanges au sein des services centraux et entre les services centra ux et les composantes se sont organisés autour de la DGS, qui assure la diffusion des informations générales nécessaires à l'appréhension des orientations stratégiques et des projets structurants de l'établissement. Ces réunions mensuelles pallient le déficit de communication inteme mais sont insuffisantes pour assurer la pleine a c culturation de tous les ac teurs et séc uriser les pratiques de chacun. Pour le comité, il serait néc essa ire de les compléter et les structurer autour de thématiques précises sur des champs plus restreints avec des interloc uteurs clairement identifiés.

L'établissement est doté d'outils de pilotage de bonne qualité (schémas directeurs immobilier, informatique, handicap, bilans sociaux, note de cadrage budgétaire) et possède une large panoplie d'applications informatiques de gestion foumies par l'Agence de mutualisation des universités et établissements (Amue), complétée d'outils développés par l'établissement sur des points précis (notamment outil de gestion des heures complémentaires). Le sentiment, justifié aux yeux du comité, de n'être «pas aussi avancé qu'il le souhaiterait en matière de données de pilotage et de tableaux de bord » 38 tient donc essentiellement à la dispersion de données de qualité dispa rate dans ces différents outils. Un travail de modemisation des outils de gestion a été lancé et une réflexion globale surl'architecture du Sl a abouti à un schéma directeur du Sl. Mais le chemin menant à une véritable urbanisation de celui-ci est encore long. Le développement, en cours, d'un infocentre collectant et fiabilisant, dans un outil unique, l'ensemble des données utilisées par l'établissement est, a ux yeux du comité, une prionité pertinente pour assurer la sécurisation des tableaux de bord de pilotage a va nt leur exploita tion. Dans cet éta blissement spatialement écla té, enc ore structurellement marqué par des tentations centrifuges et engagé dans une évolution majeure par ses projets immobiliers, l'aboutissement d'un tel projet est urgent pour objectiver les marges de manœuvre et dépasser les fragilités budgéta ires.

En matière de ressources humaines, l'établissement a établi une cartographie de ses emplois Biatss (corps/grade) dont l'enrichissement par une approche métiers et compétences a été initié fin 2017. II reste cependant à ouvrir un travail de même nature, suggéré par le rapport complémentaire de I'IGAENR, pour l'adéquation entre offre de formation et emplo is enseignants. Plus globalement, l'ensemble des procédures $\mathrm{RH}$ (dont la grille existante de rémunération des agents non titulaires n'est qu'un élément) reste pour le comité à définir dans le cadre des orientations stratégiques de l'éta blissement.

Sur la période observée, la carence en outils et données partagés a fortement limité la pertinence des échanges et dénaturé la portée réelle du dialogue de gestion. Le travail de fiabilisation des données entrepris par la nouvelle équipe, est apprécié par tous les acteurs qui regrettent, cependant, que ces échanges en

36 Masse salariale au compte financier 2015 : $170635728 € / 169040214 €$ au bilan social pour 2015. Masse salariale au compte financier 2016 : $163247619 € / 165816010 €$ au bilan social pour 2016.

37 RAE p. 57 : projet «travailler ensemble à Paris 1 Panthéon-Sorbonne ».

38 RAE p. 58. 
bilatéral entre les composantes et l'équipe de direction (VP, DGS, DAF, DRH) soient cantonnés à la seule préparation budgéta ire.

Pour autant, limité à une prévision a nnuelle mais sans suivi en exécution, le dialogue de gestion n'assure pas la visibilité en gestion qui garantirait une trajectoire stratégique, par ailleurs, et en partie pour cette raison, insuffisamment définie. Au contraire, centré sur les emplois dans la perspective exclusive des campagnes d'emplois annuelles, il s'attac he essentiellement à l'implantation des emplois créés sans analyse ra isonnée de l'ensemble des besoins au regard des ressources allouées. Dans sa forme actuelle, le comité estime donc que le dialogue de gestion, tel qu'il fonctionne aujourd'hui, conforte la tentation de reconduire l'existant plus qu'il n'incite les responsables budgétaires à une réflexion stratégique sur leurs activités dans une perspective pluriannuelle, telle que le comité propose de l'orienter.

\section{4 / LES GRANDES FONCTIONS DU PILOTAGE}

L'université Paris 1 Panthéon-Sorbonne est aujourd'hui au seuil d'une réimplantation spatiale fortement structurante pour son organisation et porteuse d'avenir pour son développement. Si la gouvemance est consciente de ces enjeux et des risques budgétaires qu'ils recèlent, c'est par une prise de conscience collective des faiblesses et des forces de l'établissement que cette étape décisive de sa riche histoire sera franchie.

\section{a / Une prise de conscience des besoins en outils de pilotage pour alimenter une réelle stratégie politique en matière de ressources humaines}

La DRH couvre les activités de paye, recrutement, gestion des camières, formation des personnels et politique sociale. Pour un volume de 2624 agents en poste (représentant une consommation de 2474,8 EIPT) en 201639, elle gère près de 5000 dossiers.

Le pilotage de la masse salariale et du schéma d'emplois est nudimentaire, limité au suivi mensuel de consommation de la masse sala riale et au suivi annuel de la consommation d'EIPT pour alimenter les tableaux budgétaires. Les informations du SIRH sont fiables mais les outils d'aide à la décision manquent: pas d'analyse fine des différents déteminants d'évolution de la masse salariale, pas de projection infra ni supra annuelle du sc héma d'emplois et de la masse salariale associée, pas d'analyse mensuelle des écarts en emplois comme en masse salariale, pas de cartographie des risques. La DRH a mivée récemment (octobre 2017) est consciente de cette faiblesse mais n'a pu encore la réduire significativement.

Les relations avec la direction des affaires budgétaires, (DAFB), se sont enrichies récemment de réunions mensuelles pour un travail plus a pprofondi et mieux doc umenté de l'exécution de la masse salariale et la DRH est déso mais associée aux dialogues de gestion budgétaire menés avec les composantes. Les échanges avec les responsables administratifs des composantes s'établissent essentiellement autour des recrutements, avec des fiches de postes soumis à l'accord préalable de la DRH mais sans évaluation préalable de leur incidence annuelle (et pluniannuelle) sur la masse salariale qui n'est calculée qu'après validation du recrutement. Les échanges avec l'agent comptable, notamment pour les opérations de paye mériteraient d'être plus noumis et d'alimenter un guide spécifique paye partagé avec tous les acteurs (responsables administratifs, DRH et AC) afin d'éviter des blocages de paye (notamment pour les Ater).

Si les premiers éléments commencent à se mettre en place, il apparaît patent au comité que la démarche qualité en matière $\mathrm{RH}$ reste entièrement à construire pour séc uriser les processus de recrutement et la gestion de la masse salariale.

Le plan de formation se concentre principalement sur 3 axes (formation aux applications de gestion, environnement métier et technologies de l'information et de la communic ation pour l'éducation) offerts au public enseignants-chercheurs et Biatss. La progression régulière du nombre de stagiaires (1 211 en 2014, 1365 en 2015, 1422 en 2016) s'accompagne d'une bonne maîtrise de la ligne budgéta ire (163 $763 €$ en 2015 pour $133142 €$ en 2016) mais trop peu d'enseignants en bénéfic ient (1 360 Biatss pour 62 enseignants bénéficiaires en 2016) ${ }^{40 .}$.

En liaison avec la DGS et le VP moyens, la DRH anime les comités techniques et est associée aux réunions du CHSCT, animées par le directeur de la prévention, de la séc unté et de l'environnement, auxquelles participent également le médecin de prévention et les assistantes sociales qui relèvent de la DRH. Le schéma directeur

$39 \quad$ Bilan social 2016.

40 lbid. 
du handicap 2014-2018 voté par le conseil académique du 27 mai 2014 fait l'objet de suivis annuels dont le demier (2016-2017), principalement centré sur les actions en faveur des étudiants, n'a pas été finalisé sur la partie ressources humaines.

\section{b / Une situation financière vulnérable, adossée à des outils de pilotage trop rudimentaires et fragilisée par les opérations immobilières en cours}

Après le déficit de 2012, l'établissement, avec l'aide ponctuelle du ministère sur certains dossiers ${ }^{41}$, s'est attaché à renouer, dès 2013, avec des exercices bénéficiaires ${ }^{42}$ en recourant, notamment, à des gels de postes pour contenir une masse salariale particulièrement dynamique. Pour autant, la situation financière de l'établissement reste extrêmement fragile avec un fonds de roulement ${ }^{43}$ inférieur au seuil de sécurité de 30 jours (22 jours à fin $\left.2016^{44}\right)$, une faible capacité d'autofinancement $(2,4 \mathrm{M} €$ en 2016 après imputation de l'annuité d'emprunt pour la rue Broca) et une rigidité budgétaire croissante ${ }^{45}$.

Cette fragilité se mesure tout particulièrement à l'aune des perspectives budgétaires que dessine l'ambitieux programme immobilier dans lequel l'établissement est engagé. Le prêt souscrit pour l'achat du bâtiment Broca 46 initialement couvert par la location des locaux à l'Institut d'administration des entreprises (IAE) 47 est désormais entièrement supporté par l'établissement. Avec l'aide ponc tuelle du ministère, l'établissement peut honorer les échéances annuelles de 1,5 M€ tout en dégageant, en moyenne depuis 2013, un solde positif d'1 M€ annuel et une capacité d'auto-financement a nnuelle de $3 \mathrm{M} €$.

Aujourd'hui, a lors que les remboursements ont déjà couvert une forte proportion des intérêts, un retra it de ce site paraît une opération financière d'autant plus défavorable qu'elle réengagerait l'établissement dans une spirale de locations immobilières pour retrouver l'espace vital qui lui est nécessaire. Or, avec la livraison des bâtiments Lourcine et Condorcet -- Aubervilliers, prévue à l'été 2019, l'établissement présente un sous financement prévisionnel de l'ordre de 8 M€ par an entre 2019 et 2020, explicité dans le schéma pluriannuel de stratégie immobilière (SPSI) ${ }^{48}$ (fonctionnement et investissement confondus). L'impasse budgéta ire est maintenue à même niveau à la livraison du site Condorcet - La Chapelle ${ }^{49}$ grâce à l'abandon total des locations. Ces prévisions, budgétairement inso utenables, ont alerté la tutelle, qui n‘a pas validé le SPSI.

Conscient de ces difficultés, l'établissement a voté un budget 2017 volontariste visant à stabiliser la masse salariale (qui représente $81,4 \%$ de ses dépenses 50 ), à réduire les dépenses de fonctionnement et à développer les ressources propres, toutes mesures qui supposent à la fois le maintien de la trajectoire en pluriannuel et un pilotage infra annuel, appuyés par des échanges réguliers avec les responsables d'enveloppes budgétaires. L'activation de l'outil d'extraction des données Sifac (outil BO) en est un préalable indispensable.

Sur la période éva luée, l'éta blissement a déjà entrepris des ac tions pour desserrer ses contraintes budgétaires. Ainsi, les dépenses de fonctionnement sont passées de 33 à 31,6 M€ entre 2012 et 2016. La proposition budgétaire pour 2017 visant une réduction de $75000 €$ seulement montre que cette action touche à ses limites, d'autant que les projets immobiliers en perspective vont nécessa irement accroître les beso ins sur cette ligne ${ }^{51}$. Le maintien en volume des rémunérations entre 2015 et $2016^{52}$ a été obtenu par la réduction de 20 EIPT $^{53}$. Bien que maintenu à $81,4 \%$ du budget au BI 2018, il est illusoire de penser poursuivre la stabilisation de

41 RAE p. 63: L'établissement a ainsi pu bénéficier d'une aide ponctuelle de la part de la tutelle lui permettant de dégager des marges de ma nœu ure sur des dossiers préc is (loyers, emplois IAE, etc.).

$422013: 0,8 \mathrm{M} € ; 2014$ et $2015: 1,2 \mathrm{M} € ; 2016 ; 1,3 \mathrm{M} €$.

43 Le fonds de roulement ne se reconstitue que très lentement : après la chute à moins de $9 \mathrm{M} €$ en 2013 , il se situe en 2016 autour de $12,7 \mathrm{M} €$, (soit près de 23 jours de fonctionnement).

44 Source : note de présentation de l'agent comptable pour le vote du compte financier de 2016.

45 L'agent comptable signale dans sa présentation au CA des comptes financiers 2015 puis 2016 la progression du ratio dépenses déca issables sur rec ettes encaissables de 97,8\% en 2015 à 98,9\% en 2016 (source : présentation au CA des comptes financiers 2015 et 2016).

46 Acheté $27 \mathrm{M} €$ avec un prêt sur 35 ans-Source RAE p. 65.

47 Créé en 1956, l'IAE est devenu établissement public administratif (EPA) en 1989, associé par convention à Paris 1. L'immeuble de la rue de Broca a été acquis par Paris 1 en 2009, grâce à un emprunt sur 35 ans, pour accueillir l'IAE. Celui-ci a finalement quitté le bâtiment en 2016, laissant à Paris 1 la charge de celui-ci, le loyer versé par l'IAE compensant jusqu'alors la charge de l'emprunt.

48 Source SPSI p. 76

49 Prévu en 2021 ma is qui aura lieu plus vra isemblablement à la rentrée 2022.

50 169,42 M€ de dépenses de personnel sur un budget de 208,17 M€.

51 Les dépenses de fonctionnement passent à 36,1 M€ au BI 2018.

52 99,488 M€ contre 99,534 M€ en 2015 - Source note de présentation du compte financier 2016 par l'agent comptable.

53 Passage de 2635 EIPTen 2015 à 2614 en 2016 - source bilan social 2016. 
la masse salariale par un recours récurrent au gel d'emplois en s'affranchissant d'une analyse fine, en infraannuel, de son exécution par composantes et services ainsi que l'y invite l'agent comptable ${ }^{54}$. Paris 1 peut prendre appui dans ce domaine sur les outils actuellement en place pour les prévisions budgéta ires ${ }^{55}$.

C'est dans le développement de ses ressources propres qu'un établissement de la taille et de la réputation de Paris 1 peut chercher de réelles marges de manœuvre notamment par la dynamisation de sa fomation continue dont la contribution est restée faible sur la période observée $\left(2,2 \mathrm{M} €\right.$ au CF 2016) ${ }^{56}$. La fondation universitaire est également un levier possible mais sa création récente ne permet pas d'en mesurer les effets budgétaires. On peut cependant raisonnablement douter qu'à elles seules, la dynamisation de la fomation continue et la création d'une fondation universitaire, suffiront à a ssurer les moyens nécessa ires a ux échéances prévues. II apparaît vital, pour le comité, dans la droite ligne des conclusions du précédent rapport d'évaluation, de mener une réflexion approfondie sur le champ des ressources potentielles notamment en matière de valorisation de sa richesse scientifique ${ }^{57}$.

Pour le comité, l'établissement doit notamment avancer plus résolument dans la mise en œuvre de la comptabilité analytique ${ }^{58}$ qui, adossée à la comptabilité réelle, est seule à même d'assurer la fiabilité de l'analyse des coûts et un pilotage optimisé de ses activités. Une réflexion préalable sur l'organisation financière de Paris $1^{59}$ sera nécessaire.

\section{c / Des projets immobiliers largement engagés, porteurs d'avenir pour l'université, dont il convient d'assurer la viabilité financière}

La direction du patrimoine pour la gestion immobilière proprement dite (exploitation, ma intenance et suivi des opérations) et la direction de la prévention, de la sécurité et de l'environnement pour le maintien des installations de sécurité et la prévention des risques se partagent la gestion du patrimoine de l'université. Leur coordination forte et dynamique, concrétisée par un secrétariat commun, est placée sous la responsabilité du chargé de mission patrimoine en lien avec le chargé de mission Condorcet.

Le schéma pluniannuel de stratégie immobilière (SPSI), validé par le CA en octobre 2017, est adossé à un système d'information du patrimoine performant ${ }^{60}$. Dispersé sur de multiples sites de dimensions variable ${ }^{61}$ et dont deux seulement sont d'importance majeure : le centre Panthéon (23 $\left.400 \mathrm{~m}^{2}\right)$ et le centre Pierre Mendès France $\left(28018 \mathrm{~m}^{2}\right)$, parta gés parfois avec d'autres établissements, le pa trimoine de l'université est hétérogène tant par ses statuts ( $83 \%$ de biens mis à disposition, $11 \%$ de biens propres et $6 \%$ de biens loués) 62 que par son état. Il est un frein à une organisation rationnelle des enseignements et de sa gestion. Le SPSI a ffiche l'ambition de réduire cette dispersion, rationaliser l'implantation des laboratoires, composantes et servic es et assurer la modemisation des outils de gestion et la professionnalisation de la fonction immobilière dans une trajectoire à 5 ans pertinente et argumentée.

Les 3 grands projets immobiliers au cœur de ce schéma, Lourcine, Condorcet -- Aubervilliers (livrés mi-2019/ mi-2020) et Condorcet -- La Chapelle (prévu pour 2022), conditionnent la réorganisation complète de

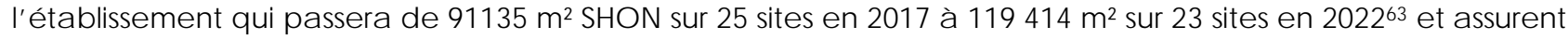
son désenga gement de locations pérennes onéreuses. Artic ulant implantations et domaines disc iplina ires, ce sc héma positionne le domaine juridique sur le quartier Latin (Panthéon, Lourcine, Sainte Barbe et René Cassin) et le domaine de l'économie et gestion à la fois sur le quartier Latin (Panthéon et Sorbonne) et à sa périphérie

54 L'agent comptable a invité à «mettre en place une projection infra annuelle d'indicateurs financiers pour détecter tout risque de déficit»- Source : présentation CF 2016 au CA.

55 Notamment fiche de coûts des diplômes et enveloppe prévisionnelle d'heures complémentaires pour chaque composante.

56 La réorientation, en 2017, de la formation continue sur des offres courtes jugées plus pertinentes semble avoir porté ses fruits dès le premier exercice (cf. entretien avec l'agent comptable) mais devra être confimée et accentuée pour que ses effets deviennent signific atifs.

57 Le rehaussement du prélèvement sur ressources propres voté par le CA ne peut être considéré comme un accroissement des ressources: pertinent pour l'équilibre du fonctionnement courant, il n'a aucun impact sur la masse globale des recettes perçues.

58 Initiée aujourd'hui sur le seul domaine de l'immobilier.

59 Structurée en 3 centres de responsabilité financières (VP moyens pour le CRB pilotage, VP CFVU pour le CRB formation et VP recherche pour la recherche), elle se décline en 19 centres financiers (dont 2 sont déclinés en départements) pour la formation, 56 centres financiers (dont 6 sont des services de thèses) pour la recherche et 14 centres financiers correspondant chacun à une direction pour les servic es centraux.

60 Source RAE page $65: 26$ sites occupés en 2017 offrant $91000 \mathrm{~m}^{2}$ SUB.

61 De $100 \mathrm{~m}^{2}$ pour les éditions de la Sorbonne à $28.018 \mathrm{~m}^{2}$ pour le centre Pierre Mendès-France - source : SPSI p. 33.

62 Source : SPSI p. 19.

63 Source : SPSI p. 58. 
immédiate (centre Mendès France et maison des sciences économiques dans le 13ème arrondissement). Les sciences huma ines sont majorita irement centrées sur les sites de Condorcet (laboratoires à Aubervilliers et 1er cycles à La Chapelle) tout en consenvant quelques implantations disséminées ${ }^{64}$, la formation continue disposant du site Broca pour pleinement se développer.

Compte tenu de l'avancement des opérations, notamment Lourcine et Condorcet -- Aubervilliers, le comité rappelle que l'assainissement de sa situation financière est une impérieuse et urgente obligation pour l'université.

64 Dans le quartier latin : Sorbonne, rue Michelet (institut art et archéologie) rue St J acques (institut de géographie). Dans le 4ème arrondissement: rue Mahler, dans le zème arrondissement; l'Institut national d'histoire de l'art, dans le 15ème a rrondissement : rue St Charles et à Nanterre, la Maison de l'archéologie et l'ethnologie. 


\section{LA RECHERCHE ET LA FORMATION}

\section{1 / LA POLTTQ UE DE REC HERC HE}

L'université Paris 1 se présente comme l'une des premières universités de recherche dans les domaines qui sont les siens, et la politique conduite depuis 2013 a eu pour objectif de renforcer cette excellence revendiquée ${ }^{65}$. L'allocation des moyens est le levier d'action le plus évident de cette politique qui s'appuie sur la qualité des relations avec les partenaires ainsi que sur la structuration des écoles doctorales et le souci, inégalement atteint, de rationalisation de la carte des équipes de recherche.

\section{a / Une structuration complexe mais globalement efficace des activités de recherche}

Les ac tivités de recherche de l'université Paris 1 sont structurées en 36 unités de recherche66, présentées en 3 familles disciplinaires : droit, science politique ; économie, gestion, maths, informatique ; sc iences humaines. Solides, les partenariats avec les organismes de recherche reposent sur une confiance réciproque. Ils concement 23 UMR C NRS (dont 1 conjointe avec I'IRD et 1 avec I'INRA), 1 UMR IRD, 1 fomation de rec herc he en évolution (FRE) a vec le CNRS, a insi qu'une USR (Maison René Ginouvès, implantée à Nanterre). Les efforts de rationalisation conduits dans l'établissement ont abouti à une réduction du nombre d'EA de 19 lors de l'évaluation précédente à 12 en 2017. Les EA restent surtout présentes en droit, management, philosophie, secteurs dans lesquels des recompositions sont encore envisageables dans le cadre de partenariats interétablissements, à l'image du rapprochement entre les chercheurs de l'EA 4101 Prism (pôle de recherches interdisciplinaires en sciences du management) et ceux de I'IAE de Paris dans la perspective de constitution d'un groupement d'intérêt scientifique (GIS) «Sorbonne recherche en management ».

L'organisation de la recherche à Paris 1 est très complexe. En effet, beaucoup d'unités associent plusieurs établissements, parmi lesquels ENS, ENS Paris-Saclay, EHESS, EPHE, AgroParisTech, Mines ParisTech, l'école d'économie de Paris, les universités Paris IV, VII, VIII, X, XII, et le ministère de la culture. Les recompositions par rapprochement avec d'autres établissements peuvent générer de l'incertitude, comme en économie dont la situation est une préoccupation importante de l'établissement ${ }^{67}$. L'éclatement des sites de recherche et le manque de locaux sont des contraintes pour les efforts de structuration. Des regroupements disciplina ires de sites et inter-établissements sont appréciés des chercheurs comme celui qui conceme les chercheurs en histoire de l'art de Paris 1 qui voisinent, rue Vivienne, avec ceux de Paris IV, Paris X et de I'INHA. D'autres rapprochements intra et inter-établissements sont prévus dans le cadre de la stratégie immobilière de l'établissement. Le site de Lourcine devrait réunir une grande partie des structures de recherche en droit en 201968. Le transfert des équipes de sciences huma ines (dont a rchéologie, géographie, histoire) à Aubervilliers dans le cadre du programme Cond orc et ${ }^{69}$ va notamment rapprocher les chercheurs en géographie physique et les archéologues et permettre l'acquisition mutualisée de nouveaux équipements, en même temps que le regroupement des bibliothèques des unités concemées. Le projet devrait également répondre aux besoins de sécurisation de l'archivage des données (bases de données, archives ora les ou visuelles, etc.). Cette logique de plate-forme et d'hôtel à projets devrait, aux yeux du comité, favoriser les synergies scientifiques, densifier «la vie des unités en concentrant leurs forces, leur donnant une cohérence par des proximités géographiques qui sont aussi des proximités thématiques, et créant, dans des ensembles pluridisciplina ires cohérents, de vastes interfa ces propic es aux fertilisa tions cro isées »70.

\section{b / Une politique qui soutient l'excellence malgré le déficit des outils de suivi des activités de recherche}

Le pilotage de la politique actuelle de recherche de l'université Paris 1 repose sur le binôme de viceprésidents, PR et MCF, prévu dans les statuts, et sur la commission recherche. Celle-ci fonctionne de manière démocratique, dans le respect des trois familles disciplinaires de l'établissement, et avec l'engagement du président au respect des décisions de la commission par le conseil d'administration. La réunion des directeurs d'unités et le collège des directeurs d'écoles doctorales, «espaces de concertation entre pairs efficaces et

65 RAE, p. 14

66 Le RAE, p. 3 indique « 36 unités de recherche dont 24 UMR » ma is le ta bleau «Structure de la recherche - juin 2017 » liste 26 UMR, 12 EA, 1 USR et 1 FRE.

67 RAE, p. 18

68 RAE, p. 64.

69 RAE, p. 8.

70 Axes stratégiques de développement de l'éta blissement. Période 2019-2023, p. 3. 
de qualité »71, permettent à deséquipes de disciplines différentes de mieux se connaître. L'éclatement spatial propre à l'établissement est, dans un certain nombre de secteurs disciplinaires, considéré comme un obstacle à la combinaison entre exc ellence disciplinaire et tra nsversalités pour des projets innovants, nationaux (Labex, ANR) ou intemationaux (programmes européens dont ERC). Cela va à l'encontre de la transversalité et l'interdisc iplina rité qui, sont fréquemment affimées comme constitutives de l'identité de l'établissement72. Le parcours doctoral «théories et pratiques de la mode", avec l'Institut français de la mode et qui conceme plusieurs unités de recherche de Paris 1 fait figure d'heureuse exception. L'établissement attend de ses assises de la recherche prévues fin 2018, qu'elles créent en inteme les conditions du renforcement des travaux interd isc iplina ires ${ }^{73}$.

Disposant d'un budget recherche sanctuarisé 74 , la commission recherche procède, au-delà de ses obligations réglementaires (répartition des grandes masses attribuées a ux écoles doctorales et aux unités de rec herche), à l'attribution des enveloppes budgétaires dédiées aux actions incitatives et à la répartition des contrats doctoraux. L'établissement a revu ses critères d'attribution, a bandonnant des «c ritères incompris ou périmés, reposant sur des notes anciennes ou des indic ateurs devenus a p proximatifs »75 au profit d'une répa rition selon des critères quantitatifs: effectifs de chercheurs et enseignants-chercheurs pour les unités de recherche et nombre de soutenances pour les écoles doctorales. En l'absence de thématiques prioritaires définies par l'établissement, les financements «politique scientifique d'établissement»(PSE), qui vont jusqu'à $25000 €$, soutiennent des projets émergents, sans exigence de construction interdisciplinaire, en contradiction avec le RAE. Les équipes bénéfic ient également de financements plus modestes au titre du bonus qualité recherche $(B Q R)$, notamment pour l'organisation de manifestations scientifiques, dès lors qu'elles assurent un cofinancement. Les préc iputs ${ }^{76}$ sont avant tout mobilisés pour le financement des réseaux scientifiques et autres GIS (une vingtaine) a uxquels partic ipe l'éta blissement.

La direction de la recherche et de la valorisation (Direval) est chargée de mettre en œuvre la politique de recherche de l'université. Son organisation en trois pôles (formations et écoles doctorales, valorisation, service financier de la recherche) en fait un lieu de ressources et de soutien pour les chercheurs et les unités de recherche, sans qu'elle dispose toutefois des moyens lui permettant d'avoir une vision d'ensemble de la vie scientifique de l'université : le service des thèses, distinct du pôle «formations doctorales », et les publications de la Sorbonne complètent l'organigramme de la Direval sans avoir de liens fonctionnels avec le directeur, tout en recevant leurs financements de la commission recherche.

La Direval gère les crédits BQR et PSE, en lien avec le CNRS pour les 6 UMR qui sont en délégation globale de gestion CNRS. Cette direction est confrontée à la diversité des pratiques disciplinaires et à la persistance d'une forte tendance à l'autonomie, renforcée par l'éloignement géographique de certaines unités. Ses moyens pour accompagner les unités dans la recherche de ressources propres sont limités: le service de la valorisation ne compte qu'une personne, spécialisée dansl'aide au montage de projets européens.

Sur la période évaluée, le comité constate que l'établissement n'a pas mis en œuvre de dispositif structuré de remontée d'information et de consolidation des données (publications, interventions dans les milieux socioéconomiques, prestations de service, etc.). Il appartient donc à chacune des unités de rec herche d'établir sa collection $\mathrm{Hal}$ et d'élaborer ses tableaux de bord, cela en l'absence de guides de procédures qui permettra ient « $d$ 'accompa gner les initiatives et $d$ ' ha moniser les pratiques, sa ns uniformiser ou caporaliser les disciplines $\gg 77$. Le comité estime donc qu'en l'absence d'un système d'information dédié à la recherche, l'établissement ne peut a voir de vision exhaustive des moyens, humains et financiers, dont disposent les unités, de la participation effective aux résea ux et GIS, des terrains de recherche et des dimensions intemationales des activités de recherche; il ne peut pas réellement engager les démarches en vue de la labellisation «HR Excellence in Research ». Autant d'éléments qui permettraient à l'université Paris 1 de mettre en avant son excellence en matière de recherche, même si celle-ci est déjà reconnue par certains classements intemationaux.

\section{2 / LA POUTTQUE DE FORMATION INITIALE ETCONTINUE}

\section{RAE, p. 16}

Cf. p. 3 et 69 du RAE.

Axes stratégiques de développement de l'éta blissement. Période 2019-2023, p. 2.

74 «Les équipes présidentielles successives ont fait le choix de la sanctua risation du budget de la recherche (hors attrition du potentiel liée au gel de postes), au moins depuis 2012. Depuis plusieurs années, le montant n'en a donc pas varié, à $3237 \mathrm{k}$. », RAE, p. 16. Affimation confimée par les bilans financiers.

75 RAE, p. 16

76 Une partie du montant des aides allouées, notamment par l'ANR, revient à l'établissement dans lequel le porteur du projet exerce sesfonctions.

77 RAE, p. 16. 


\section{a / Des formations nombreuses, fortes et originales}

L'offre de formation de l'université de Paris-l couvre essentiellement des champs disciplinaires relevant des scienc es huma ines et sociales, du droit, économie, gestion, et des arts, lettres, langues, ma is offre éga lement quelques formations en mathématiques et informatique. Cette offre est particulièrement riche car elle propose à ses 42000 étudiants 13 mentions de licence regroupant 42 parcours, 4 mentions de licence professionnelle, 63 mentions de master avec 103 parcours de M1 et 270 parcours de M2, 15 magistères, 30 préparations a ux concours et aux Meef (métiers de l'enseignement, de l'éducation et de la formation), 54 diplômes d'université et une capacité en droit.

Grâce à l'existence en son sein de disciplines proches et différentes à la fois, Paris 1 a pu introduire de la pluridisciplinarité dans les formations, ce qui constitue un réel atout pour l'insertion des étudiants et les changements qui pourront intervenir au cours de leur vie professionnelle. Cette plunidisciplinarité peut relever d'une simple coordination au sein des conseils de directeurs. II n'en reste pas moins que l'une des marques de I'université est l'existence de 16 doubles lic ences correspondant à 120 à $150 \%$ d'une licence simple. Certa ines des doubles licences sont considérées comme une classe prépara toire aux grandes écoles. Cela permet aussi une très vaste offre de masters qui rend l'université encore plus attractive à ce niveau qu'en premier cycle.

Ces formations sont éva luées et régulièrement renouvelées. L'évaluation des enseignements, mise en place à titre expérimental dans certaines UFR (celle d'économie) à partir de 2008, s'est peu à peu étendue. Cette évaluation, désomais obligatoire, se fera, à partir de 2017-2018, pour l'ensemble des licences et masters, par roulement, et chaque formation devra être évaluée au moins une fois au cours d'une même période contractuelle. Les résultats seront transmis aux comités de perfectionnement récemment généralisés et chargés de suivre chaque fomation, puis examinés par les conseils des UFR.

Le rapport AERES de 2013 c onstatait la mise en place d'espaces pédagogiques interactifs (EPI) mais regrettait une offre jugée encore trop parcimonieuse ${ }^{78}$. On ne peut que souligner le progrès incontestable accompli dans ce domaine, a utant en termes d'offres (un nombre très important de conférences, de cours, de supports sont accessibles en ligne), de formations des enseignants, de tutoriels et d'outils. Ces EPI connaissent une audience considérable selon certains directeurs de composantes avec plus de 36000 inscrits et de 10 à 11 000 attestations délivrées. Les Mooc en anglais sont récents. Une plateforme d'enseignement en ligne (Moodle) dotée de fonctionnalités étendues a été mise en place et interfacée au système d'information de Paris 1. Certaines composantes se montrent encore pour le moins réservées face à une évolution des pratiques pédagogiques qui gagne de plus en plus de terrain, et malgré le rattachement du service des usa ges numériques à la DSI, il est de plus en plus clair au sein de l'éta blissement que ce service est bien dédié à l'innovation pédagogique et non à la résolution de problèmes strictement techniques. L'établissement est membre de quatre universités numériques thématiques (économie et gestion, humanités, environnement et développement durable, juridique francophone). On notera aussi la participation des juristes au Cavej (centre a udiovisuel des études jurid iques) qui réunit 6 universités ${ }^{79}$, a c cueilla nt plus de 5000 étudia nts.

La compréhension par les lycéens des filières proposées, surtout lorsque la discipline n'existe pas dans les lycées, reste un problème. Se pose alors la question de la réorientation qui est efficace à Paris 1 , lorsqu'elle intervient très rapidement. Elle se fa it par des entretiens et des ateliers. L'enquête de l'Orive (observatoire des résultats, de l'insertion professionnelle et de la vie étudiante), "premières semaines à l'université », devrait permettre d'aller plus loin. L'université met en place des programmes-passerelles et des conventions pour fac iliter l'accès des élèves issus des CPGE, dans de nombreux domaines. Il s'ag it là d'un public-cible.

Les efforts déployés trouvent leur résultat dans la progression signific ative de la réussite depuis 5 ans. $49,1 \%$ des primo-entrants sont entrés en $L 2$ l'année suivante contre 42,7 \% cinq ans avant et $65,4 \%$ ont obtenu leur licence en quatre ans contre $57,6 \%$ cinq ans avant soit une progression de 8 points 80 .

Paris 1 a su mettre à la disposition de formations déjà reconnues et efficaces des outils et des modalités péda gogiques renouvelés, a c croissant ainsi ses performances dans ce domaine a insi que son attractivité.

\section{b / Des difficultés liées à l'attractivité}

\footnotetext{
78 AERES 2013, p. 17.

79 Université Paris 1 Panthéon-Sorbonne, université Panthéon-Assas, université Paris Descartes, université Paris-Sud 11, université Paris 13 Nord, université de Versa illes Sa int-Q uentin-en-Yvelines UVSQ.

80 RAE, p. 25.
} 
Certaines filières sont en tension en raison du grand nombre d'étudiants inscrits. C'est le domaine droit, économie, gestion qui accueille le plus grand nombre d'étudiants avec $65 \%$ de l'ensemble, les juristes représentant $29,1 \%$ de tout l'ensemble. Le domaine sciences humaines et sociales constitue $27,8 \%$ des étudiants, arts, lettres, langues 7,2 \%, mathématiques, technologies santé 3,42\% et Meef 0,1 \%, certains étudiants relevant de deux domaines.

Les conditions matérielles de la formation initiale sont difficiles en raison du manque de surfaces dont souffre historiquement Paris 1. On ne compte qu'une place pour 23 étudiants alors que la moyenne nationale est de 1 pour 11,5 ce qui oblige à des dédoublements d'amphis. Nul doute que le projet Condorcet, avec les campus d'Aubervilliers et de La Chapelle, sera un important ballon d'oxygène, même si la dispersion géographique subsistera.

L'interdic tion de rec ruter des étudiants de $M 2$ comme chargés de TD a entraîné des diffic ultés de recrutement et, pour cette année 2017-2018, certains TD ont dû se dérouler en amphithéâtres, ce qui est critiqué par les étudiants et est peu pédagogique.

L'enseignement des langues est aussi en tension, notamment en anglais, et les équipements en laboratoires de langues sont très insuffisants. Pourtant onze langues viva ntes et trois langues anciennes sont proposées ce qui est une belle richesse. Le comité estime qu'il serait aussi souhaitable de mettre en place une certification en langues. Le campus Condorcet avec une salle multimédia accolée à la bibliothèque devrait être un progrès ma is il subsistera des diffic ultés sur les a utres sites.

\section{c / Une CFVU au cœur du dispositif, organe de contrôle et d'échanges}

La commission de la formation et de la vie universita ire (CFVU) se réunit régulièrement (en moyenne une séance par mois) dans des séances qui peuvent paraître longues et chargées à certains élus puisque toutes les modifications de l'offre de fomation, des modalités de contrôle des connaissances lui sont soumises a insi que les conventions pédagogiques et intemationa les et le calendrier universitaire. Les DU font régulièrement l'objet de débats et de contrôles. Le RAE affirme que selon les statuts de Paris 1, la CFVU décide des évolutions de l'offre de fomation ${ }^{81}$. La CFVU décide également de la répartition de l'enveloppe budgétaire allouée par le CA à la formation, mais il semble que cette enveloppe dépasse les seules charges pédagogiques et englobe plus généralement les charges de fonctionnement des UFR. Cette enveloppe est pour l'instant répartie selon une clé reconduite d'année en année faute d'accord sur des critères jugés pertinents ${ }^{82}$. La participation à la CFVU est forte à l'exception toutefois de certains membres extérieurs qui n'ont, à la date de la visite, toujours pas été désignés par les organismes ou institutions représentés. Les directeurs d'UFR, des personnels de la Direction de l'enseignement et de la vie étudiante (Deve) et des porteurs de projets sont invités à participer aux séances. Ce sont eux, ainsi que les porteurs de projets, qui présentent les demandes (créations, modific ations, compléments). La commission de prépa ration des dossiers de la CFVU, composée de membres élus, souffre par contre d'une faible participation.

La CFVU de Paris 1 joue pleinement son rôle de contrôle et de validation des formationstelles qu'elles sont proposées par les composantes. Malgré le travail très important qu'elle effectue, elle ne remplit pas pleinement, a ux yeux du comité, la mission de «décider des évolutions de l'offre de fomation, »telle qu'elle est définie dans les statuts. Plus globalement, le portage institutionnel, permettant de définir par le CA ou le CAC, à l'échelle de l'établissement, la politique globale de fomation de Paris 1, apparaît au comité insuffisant. La CFVU accorde les moyens attribués par le CA aux formations mais elle n'évalue ni ne contrôle l'usage de ces moyens et leur efficacité. Pour la création ou l'ouverture d'un parcours ou d'un niveau de formation, la composante propose, la CFVU examine et, si elle en décide a insi, valide, tandis que les services centraux et la direction accompagnent. L'initiative revient donc à des composantes très autonomes et qui disposent d'une forte maîtrise de ces sujets mais ce fonctionnement gagnerait à bénéficier d'un cadrage plus net et d'objectifs globaux mieux définis, l'excellence ne pouvant résumer à elle seule, une politique. A la lumière des consta ts effec tués par le comité, ces principes et ces objectifs animent les actions conduites par les composantes en matière de formation mais faute d'un affichage clair, ils manquent de visibilité.

81 Le règlement intérieur note que «la CFVU du conseil académique et le CA examinent, sur proposition des conseils d'UFR ou d'instituts, les modifications de maquettes que ces demiers envisagent. Toute modification doit être approuvée parla CFVU du conseil académique et, en casd'incidence budgétaire, par le CA préalablement à sa mise en place. ", ce qui n'est pas tout à fait la même chose.

82 Cf. PV CFVU 24 octobre 2017. 
C'est dans le domaine de l'évaluation des enseignements qu'une politique plus volontariste a été mise en place afin d'inciter les plus réticents à la mise en œuvre de ces dispositifs testés dans certaines formations (économie, géogra phie, AES et sciences sociales).

\section{d / Le renouveau prometteur de la formation continue}

La fomation continue connaît une véritable révolution. Elle est considérée comme « un enjeu stratégique essentiel », en raison de ses missions au cœur du projet d'établissement, mais également parce qu'elle peut renforcer les resso urces propres de l'éta blissement. Alors qu'elle était fortement en crise comme le révélait un audit en 2015, une réorganisation a permis des résultats notoires avec un directeur venu du monde de l'entreprise. II y a 30 fomations diplômantes et 13 DU et les locaux sont ouverts de $7 \mathrm{~h} 30$ à $22 \mathrm{~h} 00.1300$ stagiaires y partic ipent ${ }^{83}$ dont $135 / 140$ pour le DAEU.

Il est intéressant de constater le grand nombre de modules courts très adaptés à ce type de fomation. On compte 55 formations sur trois ou quatre jours et le service répond désomais à des appels d'offre publics, ce qui avait été autrefois abandonné. Une demande prochaine d'une certification qualité de la formation continue universitaire devrait être faite.

L'apprentissage a connu un développement intéressant avec la mise en place dès 2008, de contrats professionnels (l'établissement en compte actuellement une soixantaine, et plus de 1000 apprentis) ou différentes formations dispensées en apprentissage ${ }^{84}$. Paris 1 ne dispose pas d'un CFA inteme mais travaille a vec plusieurs CFA d'lle-de-France. Le comité considère que ce travail en direction de publics variés (DAEU, formation tout au long de la vie, réfugiés) mérite d'être souligné et poursuivi.

\section{3 / LE UEN ENTRE REC HERC HE ETFORMATION}

Les études doctorales à Paris 1 associent excellence, modemité, tradition. L'université de Paris-l affiche fièrement «un docteur par jour ». La taille de l'université et ses effectifs de doctorants (en baisse continue de 4160 en 2012-2013 à 2792 en 2016-2017, dont 454 en 1ère année contre 737 en 2012-201385) ont permis la mise en place d'écoles doctora les disciplinaires, au nombre de dix : archéologie, histoire de l'art, arts plastiques et esthétique, histoire, philosophie, géographie, droit (école de droit de la Sorbonne issue de la fusion de quatre écoles doctorales en 2014), science politique, économie, management. Les modalités de fonctionnement sont très variables d'une école doctorale à l'autre, que ce soit dans les procédures de recrutement des doctorants, l'attention portée aux cotutelles, aux CIFRE ou encore au doctorat en VAE, les exigences en matière de financement de thèse ou d'encadrement (le maximum d'encadrements de thèses autorisés va rie de 7 à 15, limite qui peut encore paraître excessive). Les écarts ont toutefois été réduits avec la mise en application du déc ret de 2016 et l'instauration de comités de suivi de thèse.

Ces écoles doctorales sont ratta chées à la Direval pour ce qui relève des formations alors que le service des thèses, placé sous la responsabilité d'un professeur délégué par secteur et pour cette raison relativement a utonome par rapport à la Direval, assure les inscriptions et les soutenances des thèses. Les évolutions dans le fonctionnement des études doctorales sont globalement positives. Très apprécié par les directeurs en tant que lieu de partage d'expériences, le collège doctoral porte notamment le projet de passage du dépôt électronique (depuis 2012) à l'archivage électronique des thèses qui devrait être opérationnel en 2018-2019. Les formations transversales sont quant à elles plébiscitées par les doctorants. Ceux-ci bénéficient d'un environnement scientifique favorable en raison de l'excellence des équipes de recherche, du renom des professeurs invités accueillis, des chaires associant formation et recherche. De plus, grâce à sa gestion de l'éméritat, Paris-1 dispose d'un potentiel de professeurs émérites très utile pour les doc tora nts car il leur a p porte une aide dans des domaines spécifiques ou connexes dont les professeurs émérites ont une expérience forte. Le manque de locaux est cependant contraignant, notamment pour l'organisation des soutenances qui « demeure trop souvent problématique ${ }^{86}$, et dont les coûts sont partagés entre la Direval, les écoles doctorales, et leséquipes de recherche.

L'encadrement peut poser problème lorsqu'un enseignant-chercheur dirige un trop grand nombre de doctorants (cf. supra). Pour les réinscriptions, le passage en comité de suivi est obligatoire seulement pour la

83 Ils éta ient 1900 en 2013 ma is leur nombre était to mbé à 964 en 2014, cf. RAE p. 27.

84 On compte 1000 apprentis sur l'ensemble des champs disciplinaires (droit, économie, histoire, géographie, management, mathématiques et informatique), ne licence ou en masters, ma is également dans certains doctorats.

85 Une baisse due à la volonté d'apurer la liste des doctorants et à la limitation des durées de thèse imposée par quelques écoles doc torales. Les diffic ultés croissantes à inscrire des doc torants étrangers ont été éga lement avancées.

$86 \quad$ RAE, p. 21. 
6ème année, ce qui ne correspond pas à l'esprit des textes relatifs à la thèse. Le nombre annuel de soutenances est d'environ 350 (324 en 2016). Même si ce chiffre fait de Paris 1 un pôle majeur de formation doc torale, il est faible au regard des effectifs insc rits. Il est toutefo is diffic ile d'obtenir des données a grégées sur les abandons faute de liens solides entre les écoles doctorales et l'Orive ; il en est de même pour les données sur l'insertion professionnelle des doc teurs. Celle-ci demeure largement pensée sous l'angle des métiers de la recherche et la situation des jeunes docteurs qui n'ont pas encore été recrutés au sein des laboratoires est perçue parParis 1 comme préoccupante ${ }^{87}$.

\section{4 / UNE OFFRE DOC UMENTAIRE PRESTIG IEUSE MAIS SO US TENSION}

Comme le relèvent déjà le rapport de l'AERES de 2013 et le rapport d'autoévaluation (RAE), la politique documentaire de l'université reste extrêmement ambitieuse, à l'image du prestige et de l'excellence de l'établissement.

D'après des chiffres de $2015^{88}$, le service commun de la doc umentation (SCD) fonctionne avec un budget de $1,3 \mathrm{M} €$, la bibliothèque intenuniversitaire Cujas, a vec un budget de 2,6 M€ et la bibliothèque de la Sorbonne (BIS) a vec un budget de 3,4 M€. Rappelons que Cujas et la BIS sont rattachées administrativement à Paris 1 tout en étant des bibliothèques intenuniversitaires (avec Pa nis II pour Cujas, a vec Panis III, Sorbonne-Université, Paris V et Paris VII pour la BIS). Par ailleurs, il existe de nombreuses bibliothèques de composantes ou d'équipes de recherche.

Malgré l'exceptionnelle richesse de leurs collections, ces trois piliers de l'offre documentaire sont confrontés au défi complexe d'une hausse très importante du nombre d'usagers et d'une baisse continue des budgets. En effet, la fin du régime Cadist (crédits fléchés pour acheter de la documentation étrangère que n'a pas la BNF) a fait perdre globalement à Cujas quelque $110 \mathrm{k} €$ annuels de moyens financiers et environ le même montant pour la BIS.

\section{a / Risque de décrochage de la documentation pour la recherche}

La marge de manœuvre pour des acquisitions documenta ires est donc devenue très étroite, a vec, s'alame le RAE ${ }^{89}$, un risque réel de décrochage du niveau recherche danscertains domainesd'excellence de la BIS.

Cujas a certes pu augmenter de 40 \% ses dépenses documentaires entre 2014 et 2016, mais la BIS a dû les réduire entre 2012 et 2015 en raison de difficultés budgétaires. Ce contraste majeur entre les deux bibliothèques interuniversitaires ne s'explique pas clairement, mais les entretiens ont confimé que la subvention pourcharge de service public allouée parl'État n'a vait pas été réévaluée depuis 2009.

Dans le même temps, les charges et l'afflux de lecteurs ne cessent de croître : par exemple, la masse salariale sur ressources propres de la BIS a représenté jusqu'en 2016, $500 \mathrm{k} €$, une situation qualifiée «d'intenable »par divers interloc uteurs du comité, avant de descendre dansune fourchette de 300 à $350 \mathrm{k} €$.

Il est donc fort probable que la BIS doive continuer à limiter le nombre de contractuels sur ressources propres tout comme le nombre d'acquisitions documentaires déjà bien en-deçà du niveau minimum pour tenir son rang en temes de recherche (8000 contre 15000 ).

La réouverture en 2013 de la BIS après rénovation a provoqué un énome afflux d'usa gers (+60-70\%) tandis que les conditions d'accueil à Cujas se dégradent: 69 heures par semaine d'ouverture et quasi impossibilité de faire mieux, 1 place assise pour 29 étudiants inscrits (contre 1 pour 11 au niveau national) et $80 \mathrm{~cm}$ d'espace pour chaque lecteur dans un beau bâtiment, au cœur du quartier universitaire parisien historique, mais qui a vieilli depuis les a nnées 1950.

\section{b / Condorcet : une partie de la solution}

Au final, le défi auquel sont confrontées les bibliothèques de Paris 1 semblent relever de la quadrature du cercle.

\footnotetext{
RAE, p. 19 et 20.

RAE, p. 50.

RAE, p. 51.
} 
Une partie de la solution semble passer par la création d'un Grand équipement documentaire (GED) sur le futur campus Condorcet (prévu initialement avec une ouverture $7 \mathrm{j} / 7 \mathrm{j}$ et $24 \mathrm{~h} / 24 \mathrm{~h}$, ce qui serait une révolution) ainsi que la future bibliothèque de recherche du site Lourcine (ouverture repoussée de 2017 à 2019-2020). ॥ n'y a également qu'une seule salle pour le travail en groupe à la BIS, aucune à Cujas et qua tre salles à PierreMendès-France. Une quinza ine de salles sont prévues à Lourc ine et 40 \%à La Chapelle, une évolution positive.

Reste que l'éclatement actuel des bibliothèques et centres de documentation sur l'ensemble des sites de Paris 1 ne disparaitra pas totalement avec Condorcet. A cet égard, on ne peut que se féliciter de l'existence d'un chargé de mission de la présidence de l'université, chargé d'élaborer une vision stratégique pour les trois «piliers »doc umentaires de Paris 1.

Composante de cette vision d'avenir, la politique de numérisation et de documentation électronique est prometteuse et doit monter en puissance. Certes, il existe un plateau commun qualifié «d'exceptionnel », a limenté depuis plus de dix ans par les acquisitions des trois «piliers » qui sont consultables sur place ou à distance (69 000 titres de revues en ligne et 64000 monographies électroniques). Mais Cujas abrite au total 800000 documents en droit, économie, sciences politiques, tand is que la BIS en offre deux millions en sciences huma ines et so ciales ${ }^{90}$. 


\section{LA REUSSITE DES ETUDIANTS}

\section{1 / UN DIALOGUE EFFICACE ENTRE ETUDIANTS ETCOMPOSANTES}

L'université Paris 1 Panthéon-Sorbonne, forte de l'attractivité importante de son offre de formation, s'emploie à favoriser la réussite des tous ses étudiants. Cette volonté se traduit par un accompagnement des publics étudiants du n-1 (teminale) jusqu'à la thèse et/ou la sortie des études.

Concemant les publics entrants, l'établissement organise plusieurs joumées portes ouvertes au cours desquelles la présentation des filières se fait essentiellement par les pairs, anciens étudiants de première année pouvant répondre au mieux aux questions des lycéens. Cette volonté de bonnes relations avec le secondaire se traduit par la mission d'un des deux VP CFVU autour du continuum BAC $-3 /+3$. Le comité salue l'initiative même si cette demière, en raison d'une mise en œuvre récente, n'a pas encore pu apporter des résultats et il sera primordial d'en suivre lesévolutions.

Une description des fomations, notamment les attendus des parcours d'excellence que sont les doubles licences est disponible sur le site de l'université91. L'établissement s'est doté de nombreuses passerelles entre ses diplômes (notamment entre les doubles licences et les licences mono disciplinaires). Des réunions d'information sont organisées au début de l'année universitaire afin que les étudiants puissent se réorienter assez tôt pour ne pas perdre une année universitaire dans une réorientation. Plus globalement, d'après les étudiants rencontrés, le dialogue entre les promotions, les équipes pédagogiques et les secrétariats pédagogiques est bon et les informations circulent. L'information, bien que présente sur le site intemet n'est pas des plus faciles d'accès mais a le mérite d'être assez globale et générale pour permettre à l'étudiant de $\mathrm{s}^{\prime} \mathrm{y}$ retrouver.

Plus globalement, cette écoute des étudiants au sein des conseils de perfectionnement telle qu'explicitée durant les entretiens avec les étudiants (élus et étudiants «neutres »), les contacts directs (mails, etc.) mais aussi une évaluation des enseignements obligatoire organisée par le service universitaire numérique (SUN) pemet un dialogue constant entre les équipes pédagogiques et les étudiants, améliorant la réussite des étudiants même si les entretiens laissent apparaitre des disparités selon les composantes. L'utilisation des plateformes d'éva luation des enseignements semble fa ciliter les échang es entre étudiants et enseigna nts.

G lobalement, le comité tient à saluer cette prise en compte de la parole étudiante en vue de l'amélioration des formations. La proximité entre équipes pédagogiques et étudiants permet un lien fort entre les enseignants et leurs étudiants, favorisant la réussite de ces demiers.

L'université Paris 1 Panthéon-Sorbonne s'est dotée de régimes spécifiques d'études depuis plusieurs années mettant un accent tout particulier sur les étudiants salariés. Globalement le dispositif est bien accompagné par les enseignants même si là encore, des différences selon les composantes peuvent exister. Plus que les questions pédagogiques, ce sont les conditions d'accès aux salles pour ces publics demandant un accompagnement spécial qui posent problème.

Un suivi de l'insertion professionnelle des étudiants de master de la promotion 2014 est disponible sur le site de l'université et a été réalisé par l'Orive. Cette étude démontre une bonne insertion professionnelle des étudiants qui se disent attirés par la Sorbonne pour ces raisons. Cependant beaucoup d'entre eux ne connaissent pas l'existence de ces documents, se contentant deschiffres filière par filière.

Cette question de l'insertion professionnelle pose la question des alumni. Si des associations ou des composantes portent ces projets d'annuaire des anciens, l'établissement ne semble pas posséder une politique claire en la matière et il est encore en réflexion sur la manière de mener une politique des alumni à l'échelle de l'établissement. Un VP CFVU s'occupe plus particulièrement de cette question et il conviendra d'observer lors de la prochaine évaluation si des arbitragesont été rendus.

Sur les conditions de réussites étudiantes, la question chronique du manque de place dans les universités parisiennes, et tout partic ulièrement Paris 1, est patente. Les étudiants témoignent tous de diffic ultés à pouvo ir suivre les cours dans de bonnes conditions surtout en début d'année. Si les étudiants sont très compréhensifs sur ces questions, le comité espère que les projets immobiliers en cours permettront d'apporter une réponse à ces problèmes de locaux, notamment pour les premiers cycles.

${ }^{91}$ https://www.univ-paris1.fr/formations/ 
Au niveau des études, il s'avère que les liens entre équipes pédagogiques et étudiants sont forts ce qui permet d'accompagner plus efficacement les étudiants compensant les problèmes ma tériels que rencontre Paris 1 Panthéon-Sorbonne. Si du travail reste encore à accomplir dans la réussite étudiante, il est cependant clair que cette dynamique de dialogue contribue à améliorer l'accompagnement et la réussite des étudiants.

\section{2 / UNE PO UTTQ UE DE VIE EIUDIANTE DYNAMIQ UE, G RACE A SES ASSO C IATIO NS ET SES SERVICES}

Portée essentiellement par ses associations diverses et variées, la vie étudiante à Paris 1 est dynamique. L'établissement abrite plus de 170 associa tions qui vont des asso c iations de filières aux associations artistiques, c ulturelles ou humanitaires. Ces associations, parfois très sollic itées par les composantes, déplorent un manque de place chronique pour effectuer leurs actions ma is sont globalement au courant des différentes procédures qui s'offrent à elles (valorisation de l'engagement, demande de salle, soutien FSDIE, etc.). A ce propos les associations ont bien intégré les possibilités qu'offrait la nouvelle offre en termes de communication et n'hésitent pas à l'utiliser. Là encore le dialogue semble être réel entre les associations et l'établissement.

Ces associations sont aidées par un service de la vie étudiante intégré à la Deve. Cette aide se présente sous la forme d'un accompagnement huma in lorsqu'il est possible ou un support en temes de moyens (financier, technique, loc aux, matériels). Il est toutefois peu visible et fait pa rfois office d'appa riteur pour les étudiants ce qui, même si le service est très apprécié, n'est pas dans ses missions. Le service est globalement en tension du fait du peu de personnel alloué, sept seulement pour l'ensemble des missions étudiantes. La politique de l'établissement en matière de handicap étudiant, volontaire et ambitieuse, se heurte à un manque de moyens humains et financiers pour accomplir pleinement ses missions. En découlent un découragement et une fatigue chez les personnels qu'il conviendra de traiter. Au-delà du travail réalisé de qualité, il serait important de repenser ces missions en adéquation avec les moyens humains dont elles disposent. En l'état, seul le renforcement en personnels permettrait d'atteind re les objectifs fixés par l'éta blissement.

L'offre de Paris 1 en matière de sport, en partie mutualisée avec d'autres universités parisiennes est conséquente, variée et très professionnelle. Le sport est véritablement intégré aux études, les résultats sportifs des équipes FFSU sont a u rendez-vous et les aspects logistiques sont très bien gérés. A noter que les sports de fitness et de combat voient leur affluence augmenter. Ces pratiques nous montrent une véritable écoute des étudiants concemant les sports qu'ils veulent pratiquer. Cela démontre une politique de sport «loisir » plutôt qu'une recherche de la performance. La pratique sportive abordable, répondant aux envies des étudiants et source de bien-être fait donc clairement partie de l'ADN de Paris 1 Panthéon-Sorbonne

La politique en matière de vie étudiante est fortement axée sur l'accompagnement social des étudiants ce qui, lorsqu'on pense aux conditions de vie et d'études sur Paris, est tout à fait pertinent. Trois assistantes sociales du Crous sont à disposition des étudiants pour leur venir en aide même si ce nombre est faible pour gérer tous les dossiers (le nombre de nouveaux dossiers traités étant une preuve de la bonne connaissance du dispositif d'aide par les étudiants). Cela provoque un tumover important parmi ces personnels ainsi que des charges de travail conséquentes. II est de plus à noter que $30 \%$ des fonds FSDIE (maximum fixé par la circulaire), sont reversés à l'aide sociale.

Le partenariat avec le Crous est un partenariat solide qui pemet une politique d'aide sociale des étudiants forte et pertinente même si le manque de moyens matériels est important. Le comité salue cette politique sociale et solidaire forte conjo inte avec le Crous permettant de répondre aux besoins des étudiants.

La valorisation de l'engagement a été mise en place par l'université Paris 1 sous forme d'une gratification pouvant aller jusqu'à 0,5 points sur la moyenne annuelle. Cependant, son champ d'application très strict empêche le dispositif d'attirer davantage d'étudiants. Des disparités d'acceptation par les composantes peuvent aussi expliquer le faible nombre de dossiers (une trentaine par an). II est prévu d'assouplir les conditions d'accès à ce dispositif afin de le massifier et ainsi faire ressortir la grande vigueur du tissu associatif local ce qui est un fac teur d'attra ctivité de l'université.

Un comité se réunit régulièrement pour l'attribution des subventions FSDIE aux associations étudiantes. II a ppa rait au fil des entretiens que les associations et membres étudiants de ce comité ne s'estiment pas assez décisionnaires pour l'attribution des subventions. Malgré ce point regrettable, il apparait que l'aide aux associations est conséquente même si un assouplissement des critères est envisagé pour permettre à des associations de filière de postuler. En effet jusque-là, seuls les projets impliquant potentiellement tous les étudiants de l'établissement étaient retenus entrainant de facto une impossibilité pour les associations de filière de pouvoir déposer leurs dossiers. Le comité déplore enfin une non-conna issance du devenir du reliquat 
des fonds FSDIE. II lui semble dès lors indispensable qu'une plus grande transparence sur le devenir de ces fonds ainsi que la décision de réattribution desfonds non consommés soient mises en place.

Si les élus étudiants sont présents et bien formés à leur mission, des questionnements qua nt à leur rôle ainsi que la valeur de leur parole et prise de décision se font sentir. Une plus grande prise en compte de leurs voix semble l'une des meilleures façons de régler le problème. A ce titre si les vice-présidents étudiants possèdent quelques moyens pour exercer leurs missions et si globalement leur dialogue avec leurs principaux interlocuteurs (VP CFVU, service vie étudiante) est bon, la faiblesse des moyens qui leur sont accordés ainsi qu'un positionnement flou au sein de l'équipe politique lié à des interrogations sur leur rôle posent vraiment question. Le comité estime que le positionnement et les moyens des VPE dans un tel établissement se doivent d'être réglés a fin que les étudiants élus puissent ac complir encore mieux leurs missions.

Globalement, le comité estime que la politique de vie étudiante est ambitieuse et volontariste. Elle s'inscrit dans un partenariat fort avec le Crous et un constat juste des difficultés étudiantes en région parisienne. Les objectifs fixés lors de la demière visite de l'AERES sont en passe d'être atteints. 


\section{LA VALORISATION ET LA CULTURE SCIENTIFIQUE}

\section{1 / DES RESULTATS DE LA REC HERC HE INSUFFISAM MENTVALO RISES}

La valorisation des activités de recherche ne constitue pas un axe fort de la politique scientifique de I'université Paris 1 qui reconnaît que «la problématique des interactions avec la société civile et le monde économique n'est pas toujours au cœur de la culture de toutes les disciplines de l'université »92. Jusqu'à récemment, l'engagement de l'établissement dans la valorisation s'est seulement fa it sous l'angle du soutien a ux public ations scientifiques, et avec l'idée que la valorisation socio-économique ne concemait pas nombre de disciplines de l'établissement.

En l'absence d'une réelle prise de conscience des possibilités de valorisation en SHS, les moyens dévolus à cette mission sont limités. Elle repose uniquement sur le service valorisation de la Direval (1 personne) et sur les initiatives des chercheurs. L'établissement n'est membre d'aucune Satt (même si une collaboration a été engagée sur un projet, voir ci-dessous). Son engagement dans le développement de la valorisation est donc limité a u soutien au monta ge de projets de recherche. Les soutiens pour d'éventuelles prestations de service et le renforcement des relations avec les a cteurs soc io-éc onomiques ou culturels reposent sur le BQR et donc le co-financement par les unités de recherche. Mais les demandes dans ce domaine de l'activité scientifique émergent peu. D'autant que la Direval n'a pas les moyens humains de faire de l'information auprès des chercheurs sur ce que la valorisation pourrait être dans leur domaine. L'idée même de recenser l'activité de valorisation ne semble pas exister à Paris 1, alors qu'il y a là un potentiel significatif de ressources propres, et que la gestion des activités de valonsation des UMR est souvent faite par le CNRS (par exemple en géographie et en économie).

Globalement les conventions de transfert et d'expertise sont donc encore peu nombreuses, même si quelques projets font exception : en économie, le LabEx ReFi (Cnam, Ena, ESCP et Paris 1) sur l'évaluation des politiques de régulation financière, en statistique, le projet de plateforme Linkage mis en œuvre avec le soutien de la Satt IDF-Innov. Dans d'autres disciplines, les prestations de service continuent à relever de l'initiative et de l'intéressement individuels, sans qu'un rappel soit fait sur les obligations du chercheur en matière de cumul d'activité.

Pour le comité, la stratégie de positionnement de l'établissement sur le créneau de l'excellence et de l'insertion dans des réseaux intemationa ux s'est faite pour l'instant au détriment d'une réelle réflexion sur les apports potentiels de ses recherches au développement socio-économique ou temitorial, ou encore à la c réation culturelle. La situation pourait évoluer positivement dans les a nnées à venir. Même si elle est encore embryonnaire, la fondation Paris 1 ouvre de nouvelles perspectives en matière de relations avec les milieux socio-économiques et culturels, dans le cadre de programmes interdisciplinaires et d'interface entre recherche fondamentale et applications, y compris sous forme de financement de contrats doctoraux. Le soutien à des projets de valorisation fait aussi partie des activités prévues au sein du campus Condorcet. Paris 1 a nnonce «la mise en place prochaine d'une cellule dédiée à la prospective et aux grands programmes $\gg 3$ qui relèvera de la direction des services. Des liens forts devront être noués avec la Direval, qui souffre aujourd'hui d'un manque de moyens en matière de valorisation et d'un déficit de compétences dans le doma ine de la propriété intellectuelle ${ }^{94}$, défic it qui pourait être comblé, d'après le comité, si l'établissement intégrait la Satt IDF-Innov.

\section{2 / DES DISPOSITTFS DE DIFFUSIO N MO DESTES AU REG ARD DU POTENTIEL DE L'ETABLISSEMENT}

La relative faiblesse des liens avec le monde socio-économique dans le domaine de la recherche se traduit dans la modestie des actions de l'établissement en matière de diffusion et de développement de la culture scientifique.

Celle-ci est d'abord pensée sous l'angle du soutien à la public ation par le biais des éditions de la Sorbonne, nouvelle appellation des public ations de la Sorbonne. Leur activité dépend du renouvellement a nnuel de leur dotation (100000 $€+150000 €$ d'avances sur rec ettes) par la commission recherche de l'établissement. Elle est donc relativement limitée comparativement à d'autres presses universitaires organisées en service des a ctivités industrielles et commerciales (Sa ic) : 216 ouvrages publiés de 2012 à 2016, so it une moyenne annuelle de 35, 4 revues à comité de lecture, des revues propres à 4 écoles doctorales, et une moyenne des ventes de

RAE, p. 17

RAE, p. 16

Source : différents entretiens. 
l'ordre de $120 \mathrm{k} €$ par an depuis $2012^{95}$. Les éditions de la Sorbonne fonctionnent avec un comité de lecture qui se réunit 3 fois par an et est souverain, mais le taux de refus de publication semble faible. On peut s'interroger sur les perspectives de développement des éditions de la Sorbonne, alors que le livre scientifique connaît de réelles diffic ultés et que des universités vo isines possèd ent elles a ussi leurs Presses.

Le site web de Paris 1 est le principal canal de diffusion de la culture scientifique et de promotion des recherches de l'établissement. L'essentiel repose sur les initia tives des unités de recherche qui commencent à faire appel au service des usages numériques (SUN) pour la réalisation de vidéos. Pour l'heure la principale et modeste action de diffusion est un festival du court métrage, qui s'effectue dans le cadre d'une formation.

95 Données foumies parl'établissement (annexe recherche 18). 


\title{
VII. LES RELATIONS EUROPEENNES ET INTERNATIONALES
}

\author{
1 / RESTRUCTURATION PROMETIEUSE DE LA DIRECTION DES RELATIONS \\ INTERNATIONALES
}

La gouvemance des relations intemationales de Paris 1 est assurée par une vice-présidence et une direction des relations intemationales composée de deux services, l'un chargé de la mobilité, l'autre des coopérations intema tionales et desconventions.

Comportant vingt agents, dont un, nouvellement nommé, sera chargé plus spécialement des indicateurs d'intemationalisation, un chargé de communic ation, un secrétaire et un gestionna ire fina ncier, la DRI aspire à mener ses actions en connexion avec les autres directions et servic es de l'établissement ainsi qu'avec les directeurs des chaires et des quatre consortia d'universités fra nçaises en Roumanie, en Turquie, en Russie et en Argentine, que Pa is 1 pilote, avec les référents par pays et par réseaux, enfin a vec les délégués aux relations intemationales, enseignants-chercheurs désignés par les composantes. Dans ce cadre, il sera it pertinent que les laboratoires de recherche désignent également des délégués aux relations intemationa les a fin de faciliter le lien entre formation et recherche à l'intemational. Une commission réunit tous les acteurs des Rl quatre fois par an. Le fonctionnement et le rôle de cette commission ainsi que le déroulement des séances de travail auraient mérité d'être davantage développés dans le RAE.

Des réunions régulières entre la vic e-présidente, la directrice et les représentants des deux pôles témoignent de la bonne articulation entre l'opérationnel et le politique. La récente restructuration de la direction des relations intemationa les contribuera à la définition et la mise en place d'une politique institutionnelle articulée a utour de priorités stra tégiques.

\section{2 / UNE PO UTRQ UE RI FO NDEE SUR LES DEMANDES DES COMPOSANTES}

Les axes stratégiques et les orientations prioritaires de la politique de l'établissement ainsi que son positionnement par rapport au processus d'intemationalisation ne sont pas identifiés. Le comité regrette de ne pas avoir pu disposer d'une analyse qualitative de l'activité intemationale de l'établissement. De la lecture du RAE, il ressort une évaluation davantage focalisée sur les performances intemationales des composantes que sur une stratégie intemationale d'établissement. Des questions fondamentales telles que la mission des RI, les prionités stra tégiques, les critères qui les déterminent, les moyens mis en place pour atteindre les objectifs visés et les résultats obtenus, restent en suspens.

La juxtaposition des nombreuses activités intemationales et l'absence d'analyse critique du bilan ne permettent pas de les inscrire dans le cadre d'une politique institutionnelle. La politique affichée est essentiellement fondée sur des actions et des opportunités. Bien que l'établissement se positionne dans le contexte intemational de la fomation et de la recherche, il ne semble pas qu'il y ait coordination et articulation entre formation et recherche à l'intemational. De même, une stratégie en matière de cotutelles de thèses n'est pas clairement identifiée, l'initia tive dans ce domaine étant laissée a ux encadrants de thèses ou aux doctorants eux-mêmes.

La nouvelle dyna mique insufflée au niveau de la direction des relations intemationales, la prise de conscience des faiblesses et la forte volonté de se projeter dans l'avenir pouraient néanmoins aboutir à la réorientation de la stratégie intemationale et à la mise en place d'une politique d'établissement partagée, notamment au travers d'ind ic ateurs d'intemationa lisa tion.

Parallèlement au travail sur les indicateurs d'intemationalisation, le comité estime nécessaire de disposer d'outils pour gérer et fiabiliser les données concemant les coopéra tions à l'intemational.

De toute évidence, le nombre important de partenariats et de programmes de coopération contribue à la visibilité et à l'attractivité de l'établissement, à l'échelle européenne et intemationale. Le comité estime néanmoins important qu' une politique d'établissement so it plus clairement identifiée afin de mieux affic her et valoriser cette richesse.

\section{3 / DEVELOPPER LA MOBIUTE ET EN AMEUORER L'ACCOMPAGNEMENT}

Le RAE fait état du grand nombre d'opportunités (programmes d'échanges, doubles diplômes avec mobilité intégrée) dont peuvent bénéfic ier les étudiants de Pa ris 1 qui souhaitent effectuer une mobilité à l'étranger. II 
souligne également la nette augmentation des mobilités sortantes ${ }^{96}$ dont le nombre s'élevait à 457 en 2016 , alors qu'en 2015 la mobilité sortante concemait 356 étudiants. Pami les mesures opérationnelles et financières, prises par l'établissement pour faciliter et encourager la mobilité qui reste pour l'instant rela tivement faible, on relève une enveloppe financière plus confortable, un troisième appel de redistribution de bourses, l'encouragement du multilinguisme. Mais qu'en est-il des critiques faites par l'AERES dans son rapport d'évaluation de 2013 (p. 21) concemant l'ouverture de la mobilité à de plus larges publics notamment par «la valorisation des cursus partiellement étrangers (par exemple, les séjours Era smus) dans les filières françaises», le combat contre la «crainte répandue de sacrifier ses chances d'intégrer un $M 2$ », les mobilités des doctorants et des personnels administratifs? Aussi, la question d'un suivi qualitatif régulier des mesures incitatives se pose.

Des questionnaires en amont et en aval de la mobilité contribueraient à encourager davantage la mobilité et à a méliorer l'accompagnement des mobilités des étudiants et des professeurs invités. II est nécessaire de veiller à l'évolution du modèle de délégué aux relations intemationales, à une meilleure visibilité des éta blissements partena ires, des programmes d'études à l'étranger et des grilles de conversion des notes pour faciliter la construction des contrats pédagogiques et ne pas décourager les mobilités. Le comité souhaite que soit menée une réflexion sur le déséquilibre quant aux disciplines impliquées dans des actions à l'intemational, sur l'amélioration de l'accueil des étudiants des pays émergents qui se heurtent à des difficultés d'ordre administratif, et à l'intégration, académique et logistique, des professeurs invités, grâce notamment à la préparation en amont de leur séjour.

\section{4 / DELO CAUSATION DES FORMATIONSET INSERTIO N DANS DES RESEAUX : UNE FORCE DE PARIS 1}

La délocalisation de 31 formations, le pilotage de quatre consortia d'universités françaises en Roumanie, en Turquie, en Russie et en Argentine ${ }^{97}$, les chaires intema tiona les et l'implic ation da ns des réseaux intema tiona ux constituent sans conteste des atouts de Paris 1 qui contribuent à son attractivité et son rayonnement à l'échelle européenne et intemationale.

Le comité estime cependant qu'une procédure d'évaluation de la qualité des formations délocalisées devrait être mise en place a fin de mieux rationaliser ces coopérations et en perfectionner la structuration. De la même manière, il aurait été pertinent de montrer la plus-value de ces programmes non seulement d'un point de vue quantitatif, à savoir le grand nombre d'inscrits, mais également d'un point de vue qualitatif insistant notamment sur l'enrichissement et l'innovation pédagogiques ainsi que sur le développement d'expertise.

L'insertion de Paris 1 dans les réseaux les plus prestigieux constitue une de ses forces. Le comité regrette cependant que le RAE ne soit pas plus lisible quant à l'impact de chacun des réseaux cités sur la politique intemationale de l'établissement. Aussi, à titre d'exemple, il semble au comité peu pertinent de mettre sur un même plan ${ }^{98}$ la participation à Campus France et celle à Europaeum et Alliance qui constituent des réseaux d'universités intemationaux, a nimés par des projets communs de coopération à l'intemational.

Pami ces programmes, Alliance occupe une place privilégiée. Rassemblant depuis 15 ans, l'École polytechnique, Sciences Po, Paris 1 et l'université de Columbia, le programme Alliance entend développer désormais les coopérations entre les quatre partenaires en dépassant les coopérations bilatérales et promouvoir ainsi l'interdisciplinarité autour des thématiques transverses. Ce programme, focalisé sur les échanges d'étudiants, les co-public ations scientifiques et l'accueil de professeurs invités et des doctorants, génère une forte dynamique qui permet à Panis 1 de structurer ses activités de recherche à l'échelle nationale et intemationale.

Enfin, pour ce qui est de la projection de l'établissement dans l'a venir, il a été question, lors des entretiens, de la c réation d'une confédération européenne d'universités (Allemagne, Belgique, Espagne, France, Italie). Ce projet, priontaire pour la vice-présidence relations intemationales, permettrait de développer des liens privilégiés entre cinq universités européennes et de proposer des actions communes struc turantes. II apparaît clairement que la définition et la mise en place de cet ambitieux projet requiert une réflexion sur la politique intemationale de l'établissement et sur ses partenariats stratégiques à l'échelle nationale, européenne et intemationale.

\footnotetext{
96 RAE, p. 46.

97 Ces consortia ne font pas partie des formations déloc a lisées. RAE, p. 47 : «ils délivrent des enseignements de L et M en droit, économie, gestion et scienc es humaines et sociales ».

98 RAE, p. 46.
} 
De toute évidence, le nombre important de partenariats et de programmes de coopération contribue à la visibilité et à l'attractivité de l'établissement à l'échelle européenne et intemationale. Le comité estime néanmoins nécessaire qu'une politique d'établissement soit plus clairement identifiée a fin de mieux affic her et valoriser cette richesse. 


\section{CONCLUSION}

La force apparemment tranquille de Paris 1 repose sur la conscience d'une excellence acquise en matière de formation et de recherche, sur sa place dans l'histoire de l'université frança aise et sur des valeurs partagées au sein de l'établissement : richesse et diversité disciplinaires, ouverture intemationale, souci de l'égale représentation de toutes les composantes. C'est là ce qui constitue l'esprit et l'image, d'aucuns disent la «marque », «Panthéon-Sorbonne ».

Cette confiance en ses forces n'exclut pas la lucidité face à des pesanteurs ou des handic aps avec lesquels l'établissement a toujours su composer et qui sont la conséquence de sa taille et de sa situation au cœur de Paris: la dispersion de ses locaux, de ses structures et le poids que représentent leur entretien et leur gestion. Paris 1 a ujourd'hui reste donc fidèle à ce qui a fait sa force et sa réputation, et continue à souffrir des défic its organisationnels qui ont été constatés au fil des différentes évaluations, et tout particulièrement du manque d'outils et de procédures permettant la mise en place d'une gestion plus effic ace et mieux harmonisée. De la même manière, Paris 1 , sûre de sa réputation nationale et intemationale, peine encore à communiquer et à valoriser ses réussites.

La convic tion que l'addition ou la convergence de composantes fortes et reconnues ne sauraient constituer de réels facteurs de fra gilité, quelles que soient les conditions dans lesquelles cette convergence $s^{\prime}$ effectue, ne pousse pas Paris 1 à chercher - et donc à mettre en place - des solutions aux difficultés qu'elle a pourtant su perc evoir. La diversité disciplinaire, la richesse et la forte reconnaissance des différentes entités, mais aussi la dispersion physique en de nombreux sites, appartiennent à l'image que l'établissement a de lui-même. Cette fragmentation est d'ailleurs bien acceptée au sein de composantes qui visent, en devenant des «écoles», à renforcer leur identité et leur visibilité propres; mais elle l'est également dans un mode de gouvemance qui se veut fédérateur plus que centralisateur et que traduit le recours récurrent à la formule «Paris 1 est une Comue», qui n'est pas un simple argument justifiant la sortie d'Hésam. Exprimant, ou trahissant, la conscience de la taille et de la diversité de l'établissement et, en son sein, d'une importante autonomie de chacun, elle illustre assez bien l'identité et le projet politique de Paris 1 . Mais, du coup, si les modalités du «vivre ensemble » mises en place au fil des années favorisent une cohabitation, des collaborations intemes et des échanges, le «travailler ensemble » voire le «gérer ensemble » sont plus problématiques. Paris 1 peut se vivre et se positionner comme une Comue mais Paris 1 n'en est pas une. L'ha monisation des procédures, le dévelop pement et la modemisation d'outils communs, l'adéquation entre les moyens et les besoins, a u plus près de ces besoins, là où ils sont les plus nécessa ires, la répartition pertinente des compétences, tout ceci relève bien de la responsabilité de Paris 1 , responsabilité que l'établissement peine aujourd'hui à assurer pleinement. Des efforts de rationalisation ont été initiés ces demières années; ils doivent être étendus des outils de gestion aux procédures et aux modes d'organisation et de dialogue intemes, eux-mêmes adossés à une politique d'établissement clairement définie.

Des perspectives d'implantation immobilière, de modemisation des équipements et de collaborations nouvelles se profilent; elles permettront à Paris 1 de desserrer l'étau du manque et de la dispersion des surfaces. Mais elles exigeront aussi des moyens que Paris 1 ne poura pas uniquement attendre d'une augmentation signific ative de ses ressources, même si elle la juge légitime, ni de la manne apportée par une fondation, qui, de toute façon, sera ciblée, aléa toire et ne se fera sentirqu'à moyen ou long terme. Pour faire face à ces nouvelles charges, il lui faudra mobiliser au mieux ses ressources propres; c'est à ce prix que les perspectives qui se profilent satisferont réellement les attentes en matière d'ac cueil et d'activités.

Paris 1 poura d'autant mieux fédérer l'ensemble de ses composantes qu'elle mettra en œuvre des outils et des procédures de suivi, de gestion, de dialogue inteme, de communication, qui seront communs à tous et que chacun poura s'approprier au sein d'un cadre commun accepté par tous. Or aujourd'hui les modes de gestion de Paris 1, trop dispersés, ne sont toujours pas adaptés aux beso ins d'une université de cette taille, de cette réputation, de cette ambition. «Le temps est venu pour Paris 1 de maîtriser davantage son devenir. » Pour conjuguer au présent (et au futur) une histoire et une réputation d'excellence, la conclusion du rapport AERES de 2010 reste plus que jama is d'ac tua lité. 


\section{LES POINTS FO RTS}

- Une reconnaissance avérée de la recherche et des formations

- Une vie et une politique sociale étudiantes fortes et volontaristes

- Des partenariats forts a vec l'État, les collec tivités temitoriales, le C rous, les organismes de rec herc he

- Des projets immobiliers bien réfléchis (Condorcet, Lourcine) ouvrant des perspectives de redéploiement et de mutualisation

- L'adhésion de la communauté universitaire aux projets de positionnement stratégique de l'établissement

- Une vie démocratique bien organisée et constitutive de l'esprit de Paris 1

\section{LES POINTS FAIBLES}

- Un accueil peu satisfaisant des activités pédagogiques et scientifiques, en raison du manque de surfaces, de la dispersion des sites et de l'insuffisance des équipements

- Une fragilité financière, accentuée par l'incapacité à mobiliser efficacement les ressources financ ières et humaines

- L'absence de données fiables et partagées qui tient notamment au défaut d'urbanisation du système d'information

- Des partenariats avec le monde socio-économique et culturel qui ne font pas l'objet d'une stratégie cohérente

- L'absence de vision des enjeux relatifs à la valorisation et au tra nffert des résultats de la recherche

- Une communication inteme et exteme insuffisante

\section{LES RECOMMANDATIONS}

- Affimer plus clairement et collectivement une politique d'établissement à moyen et long termes, en partic ulier dans les doma ines de la formation et de la recherche

- Garantir la soutenabilité financière desopérations immobilières en cours et de leur exploitation

- Ha moniser et fiabiliser les procédures de gestion

- Stabiliser l'encadrement a dministratif réc emment renouvelé

- Approfondir et structurer la politique partenariale, notamment dans le cadre de la Fondation

- Donnercorpsà la stratégie d'alliance européenne déjà engagée 


\section{LISTE DES SIGLES}

A

AC

AERES

ANR

Ater

B

BI

Biatss

Bis

BQR

C

CA

CAC

Cadist

CFA

CFVU

CHSCT

Cifre

Cnam

CNRS

Comue

Condorcet

CPGE

Crous

CV

D

$D(L M D)$

DAEU

Daf

Deve

DGS

DRH

DRI

DU

E

EA

EHESS

ENC

EPA

EPHE

EPI

EPST

Erasmus

ERC

EIPT

$\mathrm{F}$

FMSH

FSDIE
Agent comptable

Agence d'évaluation de la recherche et de l'enseignement supérieur Agence nationale de la recherche

Attaché temporaire d'enseignement et de recherche

Budget initial

Bib liothéca ires, ingénieurs, ad ministratifs,

techniciens, et personnels sociaux et de santé

Bibliothèque intenuniversitaire de la Sorbonne

Bonus qua lité recherche

Conseil d'administration

Conseil académique

Centre d'acquisition et de diffusion de l'information scientifique et tec hnique

Centre de formation d'apprentis

Commission de la formation et de la vie universitaire

Comité d'hygiène, de sécurité et des conditions de travail

Convention industrielle de formation parla recherche

Conservatoire national desarts et métiers

Centre national de la recherche scientifique

Communauté d'universités et éta blissements

Projet de Cité des humanités et des sciences sociales (Paris-Aubervilliers)

Classe préparatoire auxgrandes écoles

Centre régional des œuvres universitaires et scolaires

Curric ulum vitae

\section{Doc torat}

Diplôme d'accès aux études universita ires

Direction administrative et fina nc ière

Direction des enseignements et de la vie étudiante

Directeur général des services

Direction des ressources humaines

Direction des relations intemationales

Diplôme universitaire

Équipe d'accueil

École des hautes études en sciences sociales

École nationale des Chartes

Établissement public à caractère administratif

École pratique des hautes études

Espace pédagogique interactif

Établissement public à caractère scientifique et technologique

European action scheme for the mobility of university students

(programme européen)

European Research Council

Équivalent temps plein annuel travaillé

Fondation Maison des sciences de l'Homme

Fonds de solidarité et de développement des initia tives étudia ntes 


\section{G}

$\mathrm{G}$ is

$\mathrm{H}$

Hal

Hcéres

HDR

Hésam

I

IAE

IDEX

IGAENR

Ined

IRD

I-Site

L

L (LMD)

LabEx

LSE

$M$

$M(L M D)$

MCF

Mooc

Moodle

$\mathrm{P}$

Panthéon-Sorbonne

Paris 1

PIA

PR

Pres

$\mathrm{R}$

RAE

RCE

$\mathrm{RH}$

$\mathrm{Rl}$

$\mathrm{S}$

Saic

Satt

SCD

Sciences Po

Shon

SHS

$\mathrm{SI}$

Sifac

SPSI

Sub

$\mathrm{T}$

TD

$U$

UFR

UMR

USR
Groupement d'intérêt sc ientifique

Hyper artic les en ligne

Haut Conseil de l'éva luation de la recherche et de l'enseignement supérieur

Habilitation à dinger des recherches

HESAM Université, communauté d'établissements

d'enseignement supérieur et de recherche

Institut d'ad ministration des entrep rises

Initia tive d'excellence

Inspection générale de l'administration de l'éducation nationale et de la recherche Institut national d'études démographiques

Institut de recherche pour le développement

Initia tives-Science - Innovation -Teritoires - Economie

Licence

Laboratoire d'excellence

London School of Economics and Political Science

Master

Maître de conférences

Massive open online course

Modular Object-Oriented Dynamic Leaming Environment

Université Paris 1

Université Paris 1

Programme d'investissements d'avenir

Professeur des universités

Pôle de recherche et d'enseignement supérieur

Rapport d'autoévaluation

Responsabilités et compétences éla rgies

Ressources humaines

Relations intemationales

Service d'ac tivités ind ustrielles et commerciales

Société d'accélération du transfert de technologies

Service commun de documentation

Institut d'études politiques de Paris

Surface hors œuvre nette

Sciences humaines et sociales

Système d'information

Système $d$ 'information financier a nalytique et comptable

Schéma pluriannuel de stratégie immobilière

Surface utile brute

Travaux dingés

Unité de formation et de recherche

Unité mixte de recherche

Unité de service et de recherche 
$\mathrm{V}$

VAE

VP

VPE
Validation des acquis de l'expérience

Vice-président

Vice-présid ent étudiant 


\title{
X. OBSERVATIONS DU PRESIDENT
}

\author{
A \\ unwesist \\ PANTHÉON SORBONNE
}

DIRECTION GENERALE DES SERVICES

Rèf. : CAB1-2018-09

Objet : Retour du Président sur le rapport définitif de l'HCERES

\author{
Le Président
}

à

Monsieur le Directeur du Département d'Evaluation des Etablissements

Paris, le 3 septembre 2018

Monsieur le Directeur,

Tout d'abord nous tenons à remercier vivement le Président et les membres experts du comité HCERES pour la qualité des échanges et le travail d'évaluation réalisé.

Les recommandations exprimées vont alimenter la feuille de route stratégique de l'équipe présidentielle actuelle, non seulement pour le reste de son mandat, mais pour l'avenir à plus long terme de l'Université Paris 1 Panthéon-Sorbonne. Les quelques compléments qui vont suivre montrent que les actions en cours vont déjà dans ce sens.

Tout d'abord, il est important de préciser dans la présentation de l'établissement, comme vous le mentionnez dans votre rapport, que l'Université Paris 1 Panthéon-Sorbonne est une université qui associe dès sa création plusieurs champs disciplinaires parmi lesquels il ne faut pas oublier arts plastiques et sciences de l'art, ainsi que mathématiques et informatique.

Entre le mois de janvier 2018, date de la visite sur site du comité, et la réception du rapport définitif d'évaluation, le fonctionnement de l'établissement a été très fortement perturbé entre mars et juin 2018 par le mouvement étudiant en réaction à Parcoursup et, en particulier, l'occupation du centre Pierre-Mendès-France, des avancées notoires sont à souligner. 
La politique d'Alliance que vous mentionnez en cours d'élaboration devient réalité que ce soit au niveau national avec le vote de la convention d'association par les Conseils d'Administration de Paris 1 Panthéon-Sorbonne, l'ESCP- Europe, l'INED et la FMSH ou sur le plan international avec l'accord signé pour une confédération d'établissements européens avec l'université Paris1 Panthéon-Sorbonne.

L'université a engagé des projets importants pour améliorer sa communication externe et interne, ainsi que sur le chantier des éléments de pilotage partagés avec le recrutement d'un Directeur Général Adjoint en cours qui sera à la tête d'une Direction d'Aide au Pilotage chargé, entre autre, de mettre en place la comptabilité analytique.

En matière financière, il faut rappeler, d'une part, que notre université a été pilote pour la mise en place de SIFAC GBCP, ce qui a représenté un travail considérable sur la période concernée par l'évaluation. L'établissement poursuit ses efforts sur le budget 2018, car le budget initial prévoit une diminution des dépenses de fonctionnement des Services Centraux à hauteur d'un million. D'autre part, le dynamisme des nouvelles équipes porte ses fruits: des réponses positives à des appels d'offres nous amènent des ressources supplémentaires pour 2018 en matière de formation continue et de relations internationales.

Ainsi l'université Paris 1 Panthéon-Sorbonne va poursuivre ses actions dans les années à venir en se référant aux remarques et recommandations de l'évaluation du HCERES en 2018.

Je vous prie d'agréer, Monsieur le Directeur, l'expression de ma considération distinguée.

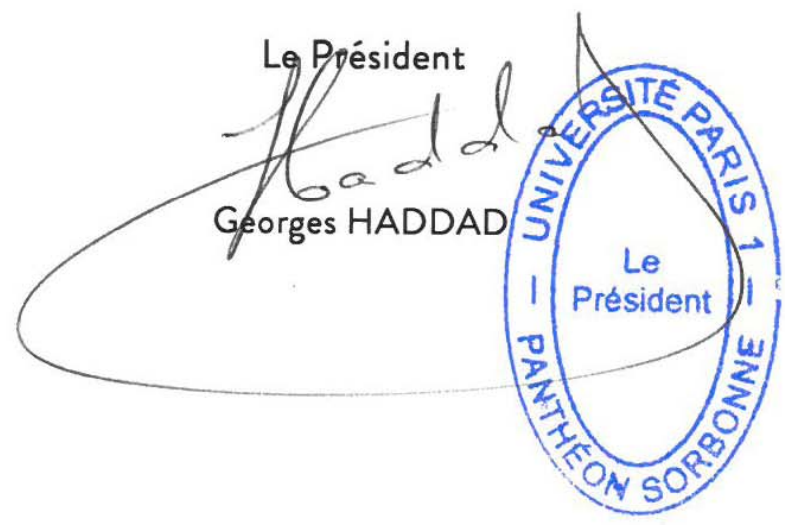

Université Paris 1 Panthéon-Sorbonne - Direction Générale des Services

12, place du Panthéon 75231 Paris Cedex 05 - Tél. : +33 (0)1 44077706 - Dgs@univ-paris1.fr 


\section{ORGANISATION DE L'EVALUATION}

L'évaluation de l'université Paris 1 Panthéon-Sorbonne a eu lieu du 30 janvier au ler février 2018. Le comité d'évaluation éta it présidé par Philippe Baudorre, professeur des universités, ancien VP CA université Bordeaux Montaigne, université de Bordeaux.

Ont participé à cette évaluation

- M. Dominique Breillat, professeur émérite de droit constitutionnel, doyen honoraire de la faculté de droit de Poitiers ;

- Mme Maryse Damaudguilhem, ancienne sec rétaire générale adjointe de l'Académie de Versailles;

- Mme Irini J acoberger, professeure des universités, vic e-présidente pour les relations intemationales, université de Stra sbourg ;

- M. Ludovic Martinet, doctorant en histoire, a ncien vice-président étudiant de l'université de Loma ine ;

- M. Nicolas Revise, joumaliste, chef de service-adjoint à l'AFP, ancien correspondant au département d'État américain ;

- Mme Raymonde Séchet, professeure des universités, ancienne vice-présidente du conseil scientifique de l'université de Rennes 2 ;

- M. Vishnu Va runyou, vic e-président de la Cour administrative suprême de Thailla nde.

Françoise Marillia, conseiller scientifique et Étienne Cazin, chargé de projet, représentaient le Hcéres.

L'évaluation porte surl'état de la coordination teritoria le au moment où les expertises ont été réa lisées. On trouvera les CV des experts en se reportant à la Liste des experts ayant participé à une évaluation par le Hcéres à l'adresse URL http://www.hceres.fr/MODALITES-D-EVALUATIONS/Liste-des-experts-ayant-participea-une-evaluation 
Les rapports d'éva lua tion du Hcéres

sont consultables en ligne : www.hceres.fr

Évaluation des coordinations territoriales

Évaluation des établissements

Évaluation de la recherche

Évaluation des écoles doctorales

Évaluation des formations

Évaluation et accréditation internationales

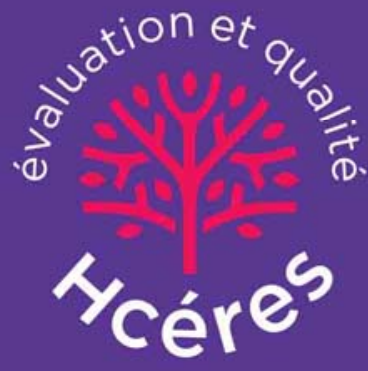

